University of Rhode Island

DigitalCommons@URI

Open Access Master's Theses

2017

\title{
Trace Detection of TATP Vapors Using a Low-Mass Thermodynamic Sensor
}

Nathaniel Gomes

University of Rhode Island, nategomes@my.uri.edu

Follow this and additional works at: https://digitalcommons.uri.edu/theses

\section{Recommended Citation}

Gomes, Nathaniel, "Trace Detection of TATP Vapors Using a Low-Mass Thermodynamic Sensor" (2017). Open Access Master's Theses. Paper 1067.

https://digitalcommons.uri.edu/theses/1067

This Thesis is brought to you for free and open access by DigitalCommons@URI. It has been accepted for inclusion in Open Access Master's Theses by an authorized administrator of DigitalCommons@URI. For more information, please contact digitalcommons-group@uri.edu. 
TRACE DETECTION OF TATP VAPORS USING A LOW-MASS

THERMODYNAMIC SENSOR

BY

NATHANIEL GOMES

A THESIS SUBMITTED IN PARTIAL FULFILLMENT OF THE

REQUIREMENTS FOR THE DEGREE OF

MASTER OF SCIENCE

IN

CHEMICAL ENGINEERING

UNIVERSITY OF RHODE ISLAND

2017 


\section{MASTER OF SCIENCE THESIS}

$\mathrm{OF}$

\section{NATHANIEL GOMES}

\section{APPROVED:}

Thesis Committee:

$\begin{array}{ll}\text { Major Professor } & \text { Otto J. Gregory } \\ \text { Everett Crisman }\end{array}$

Alan Davis

Nasser H. Zawia DEAN OF THE GRADUATE SCHOOL

UNIVERSITY OF RHODE ISLAND 2017 


\begin{abstract}
There is a growing need for a continuous monitoring system that can detect explosives in the vapor phase at trace levels. For example, triacetone triperoxide (TATP), an explosive commonly used by terrorists in improvised explosive devices (IEDs) is still going largely undetected in many densely-populated venues. No electronic trace detection system currently exists that is capable of continuously monitoring TATP vapors or its precursors. Solid-state metal-oxide sensors have demonstrated the ability to successfully detect TATP. However, to improve sensor sensitivity, the large thermal mass associated with these solid-state sensors was dramatically reduced by replacing them with extremely low mass $(25 \mu$ m diameter $)$ coil microheaters. These coil microheaters have a thermal mass that is two orders of magnitude less than the solid-state sensors and resulted in much greater signal-to-noise ratios with excellent selectivity amongst metal-oxides. These sensors were able to detect TATP, 2,4-DNT and ammonium nitrate vapors at the $20 \mathrm{ppm}$ level under varied conditions including sensor temperature, catalyst material used, inlet flow rate (sampling rate) and humidity.
\end{abstract}




\section{ACKNOWLEDGMENTS}

I would like to first acknowledge and thank my advisor, Dr. Otto Gregory for extending to me the privilege of participating in this very important research under his watchful guidance. Your patience, mentorship, and expertise have made this work an incredible learning experience for me as a student of Chemical Engineering. It has been a great privilege working alongside you on multiple projects extending back to my sophomore year in Chemical Engineering. Thank you also to my committee members Dr. Crisman and Dr. Davis for your advice and guidance throughout this work.

I would like to thank several students who were crucial to the success of this work. First, hats off to undergraduate students Andrew Rossi, Spencer Fusco, and Jonny Cummings for all of your assistance over this past year fabricating sensors, helping run various experiments and troubleshooting experimental issues. This project would not have been successful without your contributions. I would also like to thank graduate students Matt Ricci and Kevin Rivera for their assistance with sputtering operations and undergraduate student Tommy Muth for his assistance with lithography procedures. I would also like to thank former graduate student Zach Caron for his guidance in preparing me to begin this research while I was finishing my undergraduate degree.

Thanks also to Mike Platek, who has been vital in maintaining our lab equipment, troubleshooting equipment issues and making sure our labs continue to operate smoothly and safely. To the Chemistry department at URI, thank you for your time and patience preparing explosives samples for our sensor testing in such a timely manner. Thanks to the faculty and Chemical Engineering department at URI for providing me with such a 
valued education experience over the last several years. It has been a privilege learning so much from some very brilliant minds.

Finally, I owe a huge thank you to my dad for always stretching me beyond my limits and encouraging me to always strive to do the right thing, work hard, lead by example and never give up especially when work becomes difficult. To all of my friends and family, thank you for your persistent love and support throughout the course of this work. You are all a huge part of why this work became possible in my life. 
TABLE OF CONTENTS

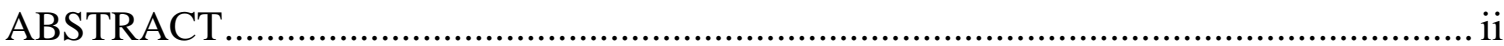

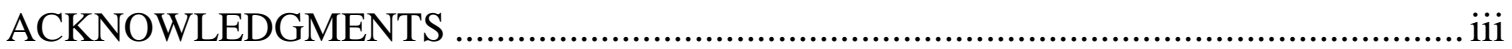

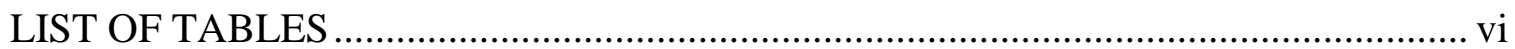

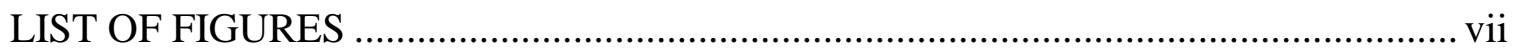

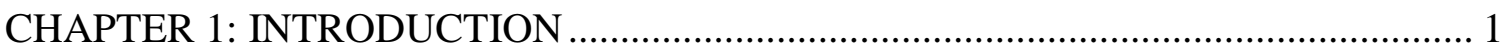

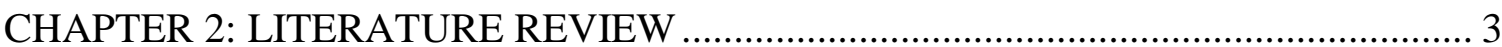

2.1 Chemical Explosives - A Dangerous Terror Weapon .................................... 3

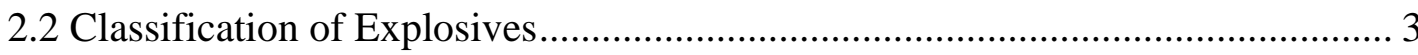

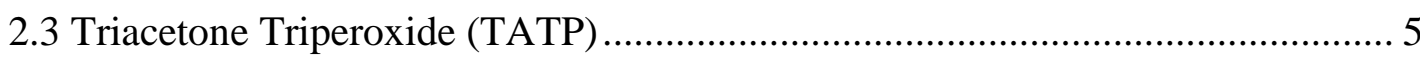

2.4 Explosives Detection Technologies .............................................................. 7

2.5 Gas-Solid Adsorption and Desorption Processes ....................................... 13

2.6 Precursor-Mediated Chemisorption ......................................................... 14

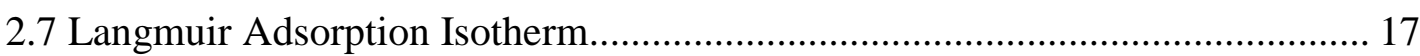

2.8 Exploiting Chemisorption to Detect TATP Vapors........................................ 18

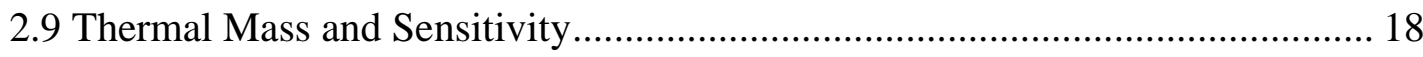

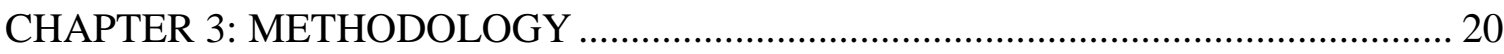

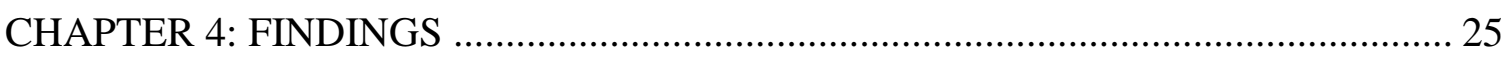

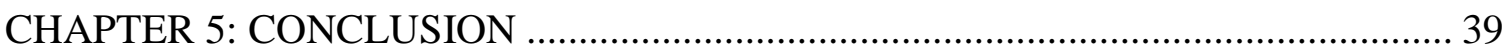

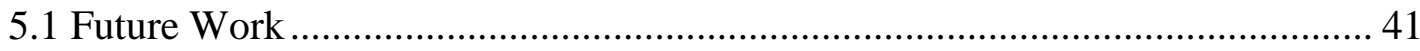

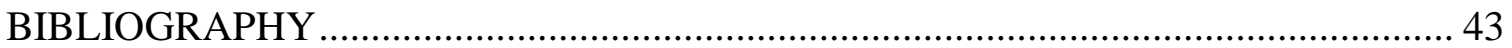




\section{LIST OF TABLES}

Table 3.1 RF sputtering conditions used to prepare the various metal-oxides used as

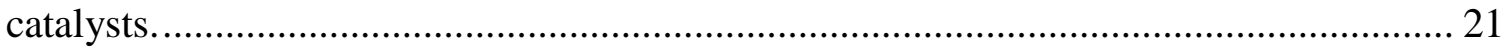




\section{LIST OF FIGURES}

Figure 2.1: Molecular structure of triacetone triperoxide (TATP). Adapted from [12]... 6

Figure 2.2: Interactions between solids and gases. Adapted from [35]. 13

Figure 2.3: Schematic of the potential energy curve for activated chemisorption. Adsorbate approaches the substrate surface (y-axis) along the curve from right to left. $E_{a d s}$ is the binding energy of the chemisorbed state, $E_{d}$ is the binding energy of the physisorbed state (and the energy barrier for desorption from the physisorbed state). $E_{a}$ is the energetic barrier to chemisorption from the physisorbed state. $\mathrm{E}_{\mathrm{des}}$ is the energy barrier to desorption from the chemisorbed state, $\mathrm{E}_{\mathrm{act}}$ is the overall activation barrier for chemisorption, and $\mathrm{E}_{\mathrm{diss}}$ is the dissociation energy of the adsorbate molecule in the gas phase.

Figure 2.4: Characteristic shapes of adsorption isotherm model systems; Langmuir (a), BET (b), and Freundlich (c) isotherms. Adapted from [21].

Figure 3.1: Schematic of the test bed used for thermodynamic sensor testing. Compressed air is fed to manual valves which are set to control the flow rates of carrier gas and TATP. Carrier gas laden with TATP flows downstream to the catalyst coated sensor and reference sensor.

Figure 4.1: Response of a $\mathrm{SnO}_{2}$ coated coil sensor exposed to 20 ppm TATP. Response (power increase) was $20 \mathrm{~mW}$ when the TATP supply was shut off. Note the slight drift in the baseline prior to the introduction of TATP. 26

Figure 4.2: Response of a $\mathrm{SnO}_{2}$ coated-coil sensor held at $75^{\circ} \mathrm{C}, 100^{\circ} \mathrm{C}, 150^{\circ} \mathrm{C}, 250^{\circ} \mathrm{C}$, $350^{\circ} \mathrm{C}, 450^{\circ} \mathrm{C}$ and $500^{\circ} \mathrm{C}$ and exposed to $20 \mathrm{ppm}$ TATP..

Figure 4.3: Arrhenius plot of the parabolic rate constant as a function of temperature for a $\mathrm{SnO}_{2}$ sensor exposed to TATP. The activation energy calculated over the temperature range of interest (Figure 4.2 "uphill" response) was approximately $35.4 \mathrm{~kJ} / \mathrm{mol}$.

Figure 4.4: Sensor selectivity to TATP for a variety of sputtered metal-oxide catalysts. $\mathrm{SnO}_{2}$ was the most sensitive catalyst to TATP vapor. Note that $\mathrm{CuO}$ exhibited a negative power change whereas all other catalysts exhibited a negative power change upon exposure to TATP. All catalysts were sputtered for 2 hours with the exception of one $\mathrm{SnO}_{2}$ sensor which was sputtered for 1 hour.

Figure 4.5: Sensor response (power change) as a function of temperature for a $\mathrm{SnO}_{2}$ catalyst coated sensor exposed to TATP, 2,4 DNT and ammonium nitrate. $\mathrm{SnO}_{2}$ coated sensor exhibited much greater selectivity for TATP relative to the nitrogen-based explosives; 2,4-DNT and ammonium nitrate. 
Figure 4.6: Responses of a $\mathrm{SnO}_{2}$ coated coil sensor to TATP as a function of volumetric flow rate. Volumetric flow rates ranging from $50 \mathrm{sccm}$ to $500 \mathrm{sccm}$ were investigated when the sensor temperature was maintained at $500^{\circ} \mathrm{C}$.

Figure 4.7: Low-mass thermodynamic sensor response to $20 \mathrm{ppm}$ TATP at $500^{\circ} \mathrm{C}$ as a function of relative humidity. An empty vessel downstream from the TATP chamber (blue curve) resulted in a lower response due to dilution effects (red curve) 36

Figure 4.8. SEM micrograph of a tin oxide coated coil after repeated thermal cycling to $500^{\circ} \mathrm{C}$ Micrograph was taken in backscattered mode.

Figure 4.9. SEM micrograph of a tin oxide coated coil after repeated thermal cycling to $500^{\circ} \mathrm{C}$. Micrograph was taken in backscattered mode highlighting the dark and light

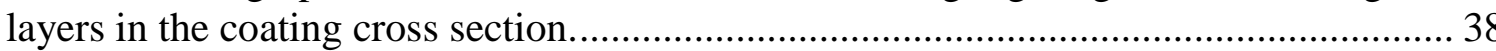




\section{CHAPTER 1}

\section{INTRODUCTION}

Terrorism involving the use of IEDs has become all too common in today's society, often with high numbers of fatalities. IEDs using explosives such as triacetone triperoxide (TATP) as the detonator for higher energy explosives or as the energetic material itself still go largely undetected in densely populated venues such as airports and subway stations. TATP has been used in a number of recent terror attacks including the 2015 Paris attack, the 2016 Brussels Airport bombing and the 2017 concert bombing in Manchester, UK. More than ever, there is a need for a continuous explosive monitoring system that can compete with a dog's nose to sniff out explosives vapors. Ideally, such an electronic trace detection system would be able to warn the public of a potential attack or be used to screen for potential threats.

Several spectroscopic methods have successfully detected TATP [1-7], but have their own limitations. These techniques require large-scale equipment that would be difficult to use in some field settings. Colorimetric sensor arrays have exhibited TATP detection as low as $2 \mathrm{ppb}$ [8], but require a one-time use swabbing technique and therefore are not suitable for continuous monitoring. One benefit to colorimetric sensor sensing, however, is its insensitivity to humidity [9]. A molecularly imprinted polymer (MIP)based sensor using differential pulse voltammetry (DPV) [10] was successfully used to detect TATP. However, it utilizes a batch process, also not suitable for continuous monitoring. Chemically modified sensor arrays [11] have been used to detect TATP at the ppq level with reasonable selectively. However, like the aforementioned techniques, it relies on batch processes/procedures that are not practical for continuous operation. 
Solid-state orthogonal sensors having a thermodynamic response as well as a conductometric response have been successfully used for the trace detection of TATP $[12,13]$. The thermodynamic platform monitors the increase or decrease in the electrical power required to maintain a constant temperature as a catalyst-coated microheater was thermally scanned. Here, the response has been attributed to heats of decomposition of the explosive molecules $[14,15]$. The conductometric platform employs a nonstoichiometric metal-oxide as the catalyst, which undergoes a resistance change when exposed to TATP and other explosive vapors $[12,13]$. These orthogonal sensors detect not only conductivity changes in the metal-oxide catalyst but simultaneously detect heat effects, which can be easily correlated with conductivity changes. $\mathrm{WO}_{3}$ based sensors developed by Warmer et al. [16] show excellent selectivity for TATP using a conductometric sensing approach but require excessively long processing times (on the order of hours). A conductometric sensor employing functionalized $\mathrm{TiO}_{2}$ nanotubes was also used by Rupashree et al. [17] to detect TATP. This approach relied on a substrate that incorporated $\mathrm{Co}^{2+}$ ions to create greater affinity towards TATP and thus induce larger conductivity changes in the substrate. However, the experimental data was too limited to prove its effectiveness in a field trial.

It has been previously shown $[14,15]$ that the detection of TATP using metaloxide sensors relies on the catalytic decomposition of the explosive as opposed to thermal decomposition suggested by Oxley et al. [18, 19]. In this work, a low-mass sensor replaced the solid state sensor described by Mallin et. al. [12]. The low-mass sensor was evaluated under varied conditions such as sensor temperature, catalyst material used, inlet flow rate (sampling rate) and humidity. 


\section{CHAPTER 2}

\section{LITERATURE REVIEW}

\subsection{Chemical Explosives - A Dangerous Terror Weapon}

Chemical explosives, upon initiation, undergo violent decomposition to produce heat, gas and rapid expansion of matter [22]. The speed at which this decomposition reaction takes place in addition to the amount of gas and heat released determines the practical effect of the explosive. If the reaction propagates through unreacted material at or below the speed of sound, it is called a "deflagration." If decomposition propagates at a speed greater than the speed of sound, it is known as a "detonation." Both of these results may be called an "explosion" by an observer. However, a detonation is a special type of explosion which is initiated by heat accompanying shock compression [22]. Before any expansion occurs, sufficient energy is released to sustain a shock wave. The shock wave propagates through unreacted material at supersonic speeds $(1500-9000 \mathrm{~m} / \mathrm{s})$ and is one of the primary physical effects responsible for explosive damage [22]. The chemical nature of the explosive, quantity used and mode of delivery all play into the success or failure of terror attacks involving explosives.

\subsection{Classification of Explosives}

Explosives may be categorized in multiple ways including their intended application in the field, their chemical structure and sensitivity to stimuli [22]. For example, military explosives have stringent criteria and must have very high performance; they must be able to be stored for decades, be transported through a wide range of environmental conditions, be stable enough to handle in battlefield conditions, 
while remaining fully functional [22]. These explosives typically are composed of the atoms carbon $(\mathrm{C})$, hydrogen $(\mathrm{H})$, oxygen $(\mathrm{O})$, and nitrogen $(\mathrm{N})$, with the nitrate groups $\left(\mathrm{NO}_{2}\right)$ that carry oxygen contributing to their performance. Common military explosives include TNT, PETN, RDX and HMX, in order of increasing performance. Plastic explosives such as C-4 or Semtex contain one or more of the military explosives above, molded with plasticizers to create moldable explosive material conveniently used for military demolitions. However, these have also been used in bombs by terrorists [22].

Commercial explosives are mostly mixtures of ammonium nitrate (AN) and fuel, replacing dynamite for most applications. AN-based mixtures offer better performance and safety at less cost. Commercial explosives do not have the high detonation velocity of military-grade explosives, but do detonate sufficiently for applications such as mining [22].

Propellants typically employ smokeless powders as the energetic material, which may include nitrocellulose, nitroglycerin, nitrotoluenes and a variety of plasticizers and burning rate modifiers [22]. Propellants are primarily intended to deflagrate, but may also be made to detonate. This is accomplished by confining the energetic material in a sealed vessel, such as in the case of a pipe bomb. Once ignited, gaseous deflagration products build up an extreme amount of pressure eventually leading to vessel rupture and an explosion [22].

Explosives are generally classified as primary or secondary explosives based on their sensitivity. A primary explosive is typically used in the detonator of an explosive device, as it is very sensitive to modest stimuli such as heat, spark or friction. Though highly sensitive, primary explosives typically are not particularly powerful. Secondary 
explosives are used in large quantities as the booster and/or main charge of an explosive device. Unlike primary explosives, secondary explosives are more stable and cannot be caused to detonate without the introduction of a significant shock. This shock requirement varies widely among secondary explosives. It may be sufficiently obtained from primary explosive detonators (in the case of "cap-sensitive" secondary explosives) or from explosive boosters [22]. Explosive boosters are required for secondary explosives that are highly insensitive, such as large-quantity commercial explosives used in bulk applications. This "explosive train" requirement is a safety feature preventing accidental detonation. Those intending to illegally use explosives typically had to steal detonators in order to effectively use them. Detonator access is tightly controlled by many governments and law enforcement agencies. However, terrorists have found ways around this by employing homemade explosives such as TATP as the detonator for explosive devices [22].

\subsection{Triacetone Triperoxide (TATP)}

Triacetone triperoxide (TATP, Figure 2.1) is an organic peroxide explosive of choice in many improvised explosive devices (IEDs) used in terror attacks because it can be synthesized from common, inexpensive starting materials. TATP is extremely sensitive and thus is generally classified as a primary explosive. Synthesis and handling is extremely dangerous, as the chance for accidental detonation is greater than that of more stable commercial explosives. It is synthesized using hydrogen peroxide $\left(\mathrm{H}_{2} \mathrm{O}_{2}\right)$ and acetone $\left(\mathrm{C}_{3} \mathrm{H}_{6} \mathrm{O}\right)$ in the presence of a strong acid. The raw materials are mass produced for many other commercial uses, allowing them to be easily obtained by 
terrorists. TATP is a very powerful explosive which produces about $88 \%$ of the blast strength that TNT produces by mass. Consequently, TATP can be used both as an unstable primary explosive (initiator) while also being powerful enough at the right concentrations to be used as a detonable secondary explosive [22].

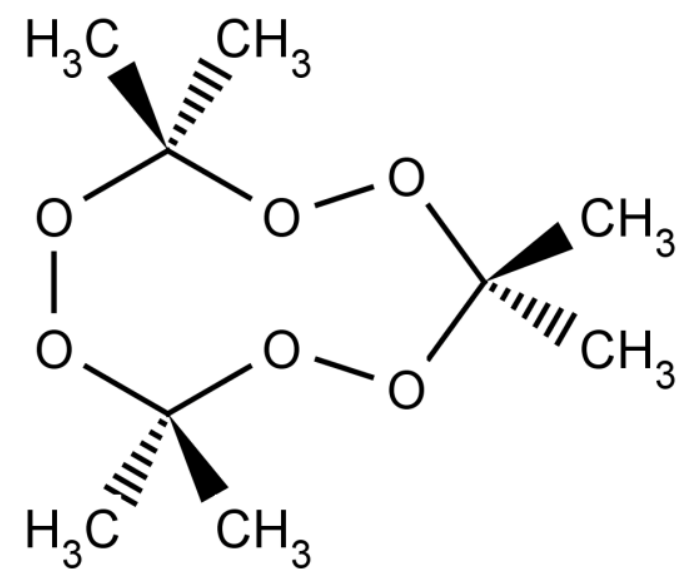

Figure 2.1: Molecular structure of triacetone triperoxide (TATP). Adapted from [12].

Commercial and military explosives are more ideal in terms of performance and safety over TATP. However they are not nearly as easy to obtain and may also contain chemical "tags" - volatile organics which may be used for forensic purposes to track down the original source of the explosive's production [23].

Terrorist groups are getting more creative with attacks employing IEDs with peroxide explosives. By using homemade explosives such as TATP or HMTD (another peroxide-based explosive), they avoid detection on all fronts - from the time they purchase the raw materials for synthesis to when they prepare to detonate the explosive. TATP is not among the highest-performing explosives that exist, but is still incredibly dangerous and is responsible for many incidents over the last several years which have killed and injured many people: 
On August 9, 2001 a suicide bomber outside of a pizza restaurant in Jerusalem detonated a TATP explosive belt, killing 15 people and injuring in excess of 90 people [1]. Later that year, Richard Reid failed to detonate a "shoe bomb" by lighting a TATP detonator on an international flight. On July 7, 2005, bombings of the underground subway and bus systems in London took place using TATP detonators, killing 52 innocent people plus 3 suicide bombers [1]. On October 1, 2005, a University of Oklahoma student killed himself using a TATP IED [1]. In 2006, a man was killed and another severely injured in a TATP manufacturing accident in Texas City, Texas [1]. German police in September 2007 disrupted a terrorist cell linked to an Islamic Jihad militant group, which had amassed 1,500lbs of hydrogen peroxide to be used in preparation of future IEDs [1]. TATP was detected on two workers at the Oskarshamn nuclear power plant in 2008 [1]. Afghan immigrant Najibullah Zazi was arrested in September 2009, suspected of being connected to attempted synthesis of TATP - he pled guilty to those charges in early 2010 [1]. On Christmas day, 2009, Umar Farouk Abdulmutallab attempted to detonate an explosive device made with PETN initiated by TATP while onboard an international flight from Amsterdam to Detroit, MI [1]. More recently, TATP was used in the 2015 Paris terror attack, the Brussels Airport bombing in Belgium in 2016 and the Manchester, UK concert bombing in 2017.

\subsection{Explosives Detection Technologies}

The most effective method currently used today for explosive vapor detection is through the use of trained dogs, capable of sniffing out explosives vapors at extremely low concentrations, following the scent to the source. There are no detectors in place today which can rival the ability of trained dogs to perform this same task. However, 
dogs do have their shortcomings; they require rest and can tire over time, losing their sensitivity. They have relatively short attention spans and are easily distracted. Other chemical vapors can also mask the scent of the explosive and result in false-negative (missed) explosive detection [24].

Various competing technologies have been developed over the last few decades which focus on detecting explosives by exploiting the characteristics of their unique physical and chemical properties. These properties determine how effective a given sampling technique or detection method will work. For example, methods intending to detect explosives in the vapor phase will struggle to detect explosives with extremely low vapor pressures such as RDX or PETN. Those with high vapor pressures (especially higher than TNT), such as ammonium nitrate, nitroglycerine, dinitrotoluene (DNT) and TATP will be more prone to vapor detection methods. Vapor pressure plays a large role in distinguishing some explosives from other chemicals. For example, retention time is sometimes influenced by vapor pressure and is an important characteristic for techniques employing gas chromatography for detection. Explosives with low vapor pressures are instead detected through the use of particulate detection systems [24].

Explosives detection may take place through the use of explosives trace detection (ETD) systems or through bulk detection technologies. ETDs may detect explosives in the vapor phase by directly sampling from the air or by trace amounts of particulate residue on inspected objects. Both cases require rapid chemical detection techniques for identification of explosive molecules. ETDs may successfully detect via exposure to trace amounts of explosive vapors and are more effective with the use of preconcentration $[12,13]$ or other effective sampling techniques to increase the exposure 
of explosives to detectors. Thus, sampling techniques are another aggressive area of research. Some examples of trace detection technologies include ion mobility spectrometry (IMS), surface acoustic wave (SAW), chemiluminescence, amplifying fluorescent polymers, and mass spectrometry [24].

IMS detects the characteristic mobility of explosive ions in an electric field under atmospheric pressure and is the most used field trace detection technology for explosives at airport checkpoints and by law-enforcement. IMS has been intensively developed over the last 20 years and offers high sensitivity, simple instrumentation, low cost, broad application and real-time monitoring capabilities. It is easy to operate and may be used for vapor or particulate detection. This makes IMS one of the first choices for field ETDs as IMS detectors claim to be able to detect TNT, RDX, PETN, NG, and TATP at very low levels. However there is room for false-positive detection using IMS, as the presence of several substances such as food, oil, etc. may trigger a false-alarm. The technology relies on dry air flow through the ion drift region and ambient humidity can alter the moisture content of this region. The increase in moisture can alter the mobility of ions of interest passing through the device [24].

Surface acoustic wave (SAW) technologies consist of interdigital transducers deposited on piezoelectric crustal surfaces, where the energy of SAWs are conserved and surface perturbations lead to significant changes in surface acoustic wave properties. Sensors have been developed typically consisting of a periphery circuit, a SAW detector and a sensitive thin film deposited on the detector surface. The thin film can strongly absorb specific gases and this absorption changes the sensor's oscillation frequency. This change is measured and used as the basis for identification of the gaseous content. Some 
techniques have employed gas chromatography in front of the detector (SAW-GC) to separate and identify multi-component gases. This technique is particularly useful for detecting a wide range of volatile organic compounds (VOCs) and semi-volatile organic compounds (SVOCs) [25].

Chemiluminescence (CL) detection techniques rely on the generation of electromagnetic radiation (ultraviolet, visible, or infrared) as light by the production of an electronically excited species from a number of reactants, which releases light in order to revert to its ground state energy. CL has advantages of high sensitivity, safety, rapid and simple assay and controllable emission rate. It also has excellent selectivity when compared to high-speed gas chromatography (GC). However, it suffers from poor reproducibility and long observation times. For explosive detection, it is limited only to nitrogen-containing explosives and cannot be used for peroxide explosives [26].

Amplifying fluorescent polymer (AFP) technologies measure an increase or decrease (quenching) in fluorescence intensity that occurs when a single molecule of analyte interacts with a single fluorophore. When thin films of AFP materials absorb a photon of light, excited state electrons (excitons) are able to migrate efficiently along the conjugated polymer backbone and between adjacent polymer chains. The binding of TNT molecules (or related nitroaromatics such as 2,4-DNT) to these materials result in a dramatic reduction in the emission intensity and is proportional to the mass of quencher adsorbed by the films. This is measured by sensor systems. This technique is limited to detection of nitroaromatics, particularly TNT used in landmines [27].

Mass spectrometry (MS) in various forms (quadrupole, ion trap, time-of-flight, tandem-based and hybrids) have been used for detection of energetic materials due to its 
selectivity, sensitivity, reliability and speed [28]. MS is a proven technology and a major contributor to the technology that makes it possible for experts to monitor and detect explosives. MS classifications of compounds are performed by ionization followed by separation based on mass-to-charge ratios $(\mathrm{m} / \mathrm{z})$. The disadvantages of this technique are that it is quite costly and the instrumentation required is large in size. However, some progress has been made in attempting to miniaturize this technology [29-33].

Bulk detection techniques utilize some of the physical and/or chemical characteristics of a macroscopic amount of explosives and include nucleus-based and image-based technologies. Nucleus-based technologies detect at the atomic level and use high-energy ionizing radiation source or nuclear resonance for the detection process. Detectors measure radiation (usually gamma-rays) emitted from target materials and the output information reveals its atomic constituents. These technologies in general do not directly detect explosives, but rather indirectly detect through the presence of common atoms found in explosive materials such as nitrogen, carbon, oxygen and hydrogen in varying ratios. High-energy neutron sources damage the cells of the human body and thus cannot be used to search people, but may be used for searching cargo, trucks, and luggage. Examples include thermal, fast neutron, and pulsed fast neutron technologies. Nuclear quadrupole resonance technology can also be used for atomic bulk detection, which excites nuclei with quadrupole properties (such as nitrogen) using radio frequency radiation, measuring the return signals from the target to determine whether or not explosives are present. However, this technique is limited to nitrogen-containing explosives and cannot detect peroxide-based explosives [24]. 
Imaging techniques for bulk detection include various X-ray technologies (transmission, dual-energy, computer tomography, backscatter) and millimeter wave imaging. These techniques do not attempt to detect the chemical constituents of explosives, but instead look for the shape and size expected of explosive devices, as well as concealed weapons like knives or guns. The effectiveness of this technique depends on human interpretation of the produced images and has also raised concerns over privacy and health-related issues [24].

ETD technologies clearly provide the best chance for detection of TATP over bulk techniques. The goal for ETDs is to selectively and sensitively detect explosives quickly with a low false-alarm rate in order to provide earlier warning and improved safety for the public. No technology is $100 \%$ reliable and false alarms are always possible based on the limitations of various technologies. Ideally, detectors should be easy to use, portable, reusable and rugged. They should also have a relatively high throughput rate by minimizing the time required for scanning a particular item for trace amounts of explosives. Sensors have the best chance for meeting these needs, as they are low cost, portable, and may operate continuously with minimal maintenance [34]. Sensors contain an active material which will selectively interact with target explosives by physical phenomena which may be transduced to a measurable property. Sensors have the benefits of real-time, selective environmental monitoring but may struggle with sensitivity. There is also potential to utilize a variety of sensors as an array in order to scan for multiple target explosives at once. Common sensors include conductometric sensors (measures conductivity changes in the material upon exposure), optical sensors (chemiluminescence, etc.), mass sensors (surface acoustic waves, microcantilevers, etc.), 
colorimetric sensors (color changes upon exposure) and calorimetric sensors (detecting heat-related effects).

\subsection{Gas-Solid Adsorption and Desorption Processes}

Three fundamental solid-gas interactions (Figure 2.2) are possible in adsorption processes: physisorption, chemisorption, and non-reversible reactions with the surface [35]. Physisorption occurs due to weak, longer-range Van der Waals forces and results in no dissociation of the adsorbate. Enthalpies of physisorption are very small, at approximately $0.2 \mathrm{eV} /$ molecule [36]. Chemisorption, or chemical adsorption, is a much stronger interaction resulting from chemical bond formation between (usually) dissociated gas molecules and the solid surface, with charge transfer taking place [35]. However, covalent bonds may also take place [20]. Chemisorption enthalpies have larger magnitudes around 2eV/molecule [37]. The distinction between physisorption and chemisorption by energies alone is not a sharp distinction, but it is conventionally accepted to be at an adsorbate-substrate binding energy of about $0.5 \mathrm{eV} / \mathrm{molecule}$ (note that $1 \mathrm{eV} / \mathrm{molecule}=23.060 \mathrm{kcal} / \mathrm{mol}=96.485 \mathrm{~kJ} / \mathrm{mol}$ ) [20]. Non-reversible reactions typically lead to the formation of new compounds and are generally interactions that are avoided in gas sensor operation altogether [35].

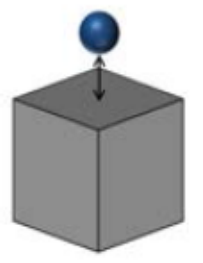

(a) Physisorption

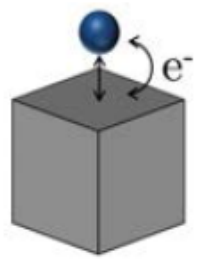

(b) Chemisorption

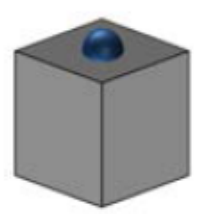

(c) Non-reversible reaction

Figure 2.2: Interactions between solids and gases. Adapted from [35]. 
Physisorption is usually only detected when chemisorption is completely absent and when the substrate temperature is low, such as in the case of low-temperature adsorption of noble gases on metal surfaces [20]. A typical example of chemisorption is the adsorption of metal atoms onto other metal and semiconductor surfaces at high temperatures. The strong interaction - either through covalent or ionic bonding changes the adsorbate chemical state and may cause dissociation and formation of new adsorbate species. The substrate structure is also changed, ranging from relaxation of interlayer spacing of the top substrate layers to substrate reconstruction, which involves complete rearrangement of the top layer atomic structure. In the case of surface reconstruction, thermal activation is required [20].

\subsection{Precursor-Mediated Chemisorption}

There are multiple activation energy barriers associated with any given adsorption process, especially with the case of precursor-mediated chemisorption. Precursormediated chemisorption may occur in the form of activated chemisorption (shown in Figure 2.3), or non-activated chemisorption. The difference for non-activated chemisorption is that the activation energy barrier between physisorbed and chemisorbed states $\left(\mathrm{E}_{\mathrm{a}}\right)$ is significantly lower (less than $\mathrm{E}_{\mathrm{d}}$ ), and actually takes place entirely below the $\mathrm{x}$-axis (initial energy state).

This difference affects the temperature dependence on the "sticking coefficient"

for adsorbing species [20]. The sticking coefficient, $s$, is the ratio of the adsorption rate to the impingement rate of adsorbate gas molecules. At increased temperatures for the 
activated adsorption case $\left(E_{a}>E_{d}\right)$ the sticking coefficient increases, while for nonactivated adsorption $\left(E_{a}<E_{d}\right)$ the sticking coefficient decreases with increased temperatures. This coefficient acts as a correction factor which properly defines the adsorption rate for a system, given a flux of gas molecules, I, impinging on a surface from the gas phase; that is, the rate of adsorption $r_{a}=s^{*} I[20]$. The greater the sticking coefficient, the greater overall adsorption rate to the surface given constant flux.

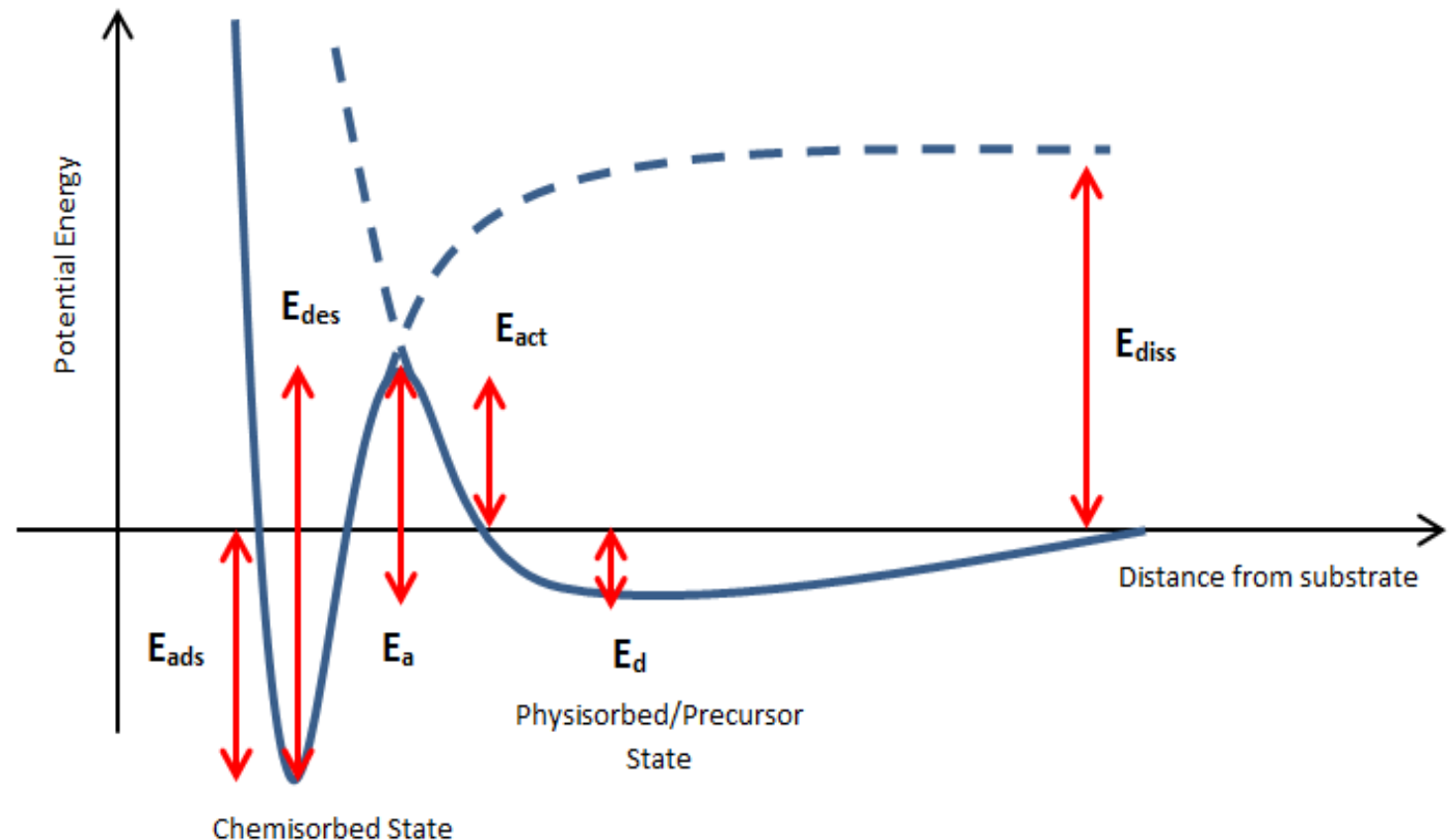

Figure 2.3: Schematic of the potential energy curve for activated chemisorption. Adsorbate approaches the substrate surface (y-axis) along the curve from right to left. $\mathrm{E}_{\text {ads }}$ is the binding energy of the chemisorbed state, $E_{d}$ is the binding energy of the physisorbed state (and the energy barrier for desorption from the physisorbed state). $\mathrm{E}_{\mathrm{a}}$ is the energetic barrier to chemisorption from the physisorbed state. $\mathrm{E}_{\text {des }}$ is the energy barrier to desorption from the chemisorbed state, $\mathrm{E}_{\text {act }}$ is the overall activation barrier for chemisorption, and $\mathrm{E}_{\mathrm{diss}}$ is the dissociation energy of the adsorbate molecule in the gas phase.

Figure 2.3 depicts a typical activated chemisorption case, but may also be used to depict other types of adsorption pathways. For example, adsorbate may only physisorb to a surface through Van der Waals interactions and not overcome the activation energy 
barrier required for chemisorption $\left(\mathrm{E}_{\mathrm{a}}\right)$. It could instead desorb back along the physisorption curve away from the influence of the surface, provided it does not have the energy required to overcome $\mathrm{E}_{\mathrm{a}}$. If however the adsorbate molecule is able to overcome this larger energy barrier, chemisorption will take place through either covalent or ionic bonding behavior with the surface - a much larger binding energy than physisorption energies. The distance from the surface to the adsorbate in the chemisorbed state is the adsorption bond distance. Note that moving along the curve in the opposite direction (left-to-right) is the desorption curve.

The energy required to desorb adsorbate molecules from the surface depends on the nature of how it is adsorbed. For activated chemisorption (Figure 2.3), it can be seen that the energy required to desorb ( $\left.E_{\text {des }}\right)$ is equal to the sum of the chemisorption activation energy $\left(\mathrm{E}_{\mathrm{act}}\right)$ and chemisorption binding energy $\left(\mathrm{E}_{\mathrm{ads}}\right)$. For non-activated chemisorption, $\left(\mathrm{E}_{\mathrm{act}}\right)$ occurs completely below the zero-energy line, resulting in $\mathrm{E}_{\mathrm{des}}=$ $\mathrm{E}_{\mathrm{ads}}$; in this case, only the chemisorption binding energy needs to be overcome in order to desorb. For physisorption, the desorption energy is simply $E_{d}$, shown in Figure 2.3. $\mathrm{E}_{\mathrm{diss}}$ is the energy required to dissociate a given adsorbate molecule still in the gas phase away from the influence of the surface (if applicable). This energy down payment is unfavorable - however it may be overcome through a chemisorption pathway if using an appropriate surface to catalyze the dissociation behavior. Following the adsorption curve to the "precursor state" shown in Figure 2.3, adsorbate molecules may still be intact at a physisorbed location. Upon overcoming the $\mathrm{E}_{\mathrm{a}}$ activation barrier, the adsorbate may then dissociate into two (or more) adsorbate species which may then chemisorb individually by bonding tightly to the surface. This is a more energetically- 
favorable pathway to a similar dissociative result and a classic example of heterogeneous catalysis [38]. Chemisorption coverage at the surface may be well-represented by the Langmuir Isotherm [39].

\subsection{Langmuir Adsorption Isotherm}

The Langmuir adsorption model $[20,21,38,39]$ makes the following assumptions: Adsorption is limited by monolayer coverage, all adsorption sites are equivalent and only one molecule can reside in a given adsorption site. Chemisorption binding energies are strong enough to fulfill Langmuir's adsorption criteria [39]. The Langmuir isotherm is a characteristic plot of surface coverage versus concentration of adsorbate, which is shown as curve (a) in Figure 2.4:

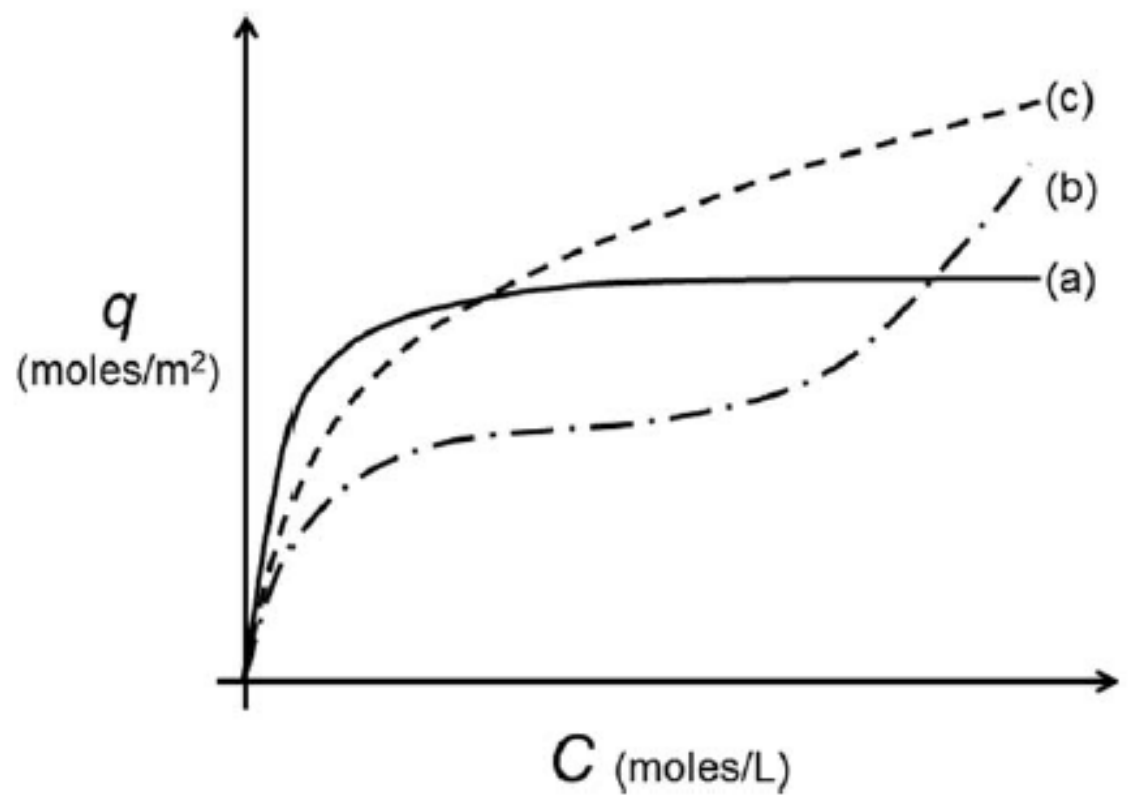

Figure 2.4: Characteristic shapes of adsorption isotherm model systems; Langmuir (a), BET (b), and Freundlich (c) isotherms. Adapted from [21]. 
The BET (Brunauer-Emmett-Teller) isotherm (b) is a shape that is more characteristic of multilayered adsorption systems [39], while the Freundlich isotherm (c) better depicts monolayer adsorption onto heterogeneous surfaces. The Langmuir adsorption model's curve shape was highly representative of some of the early sensor responses obtained for the new low-mass sensors described in this work. Noting this characteristic curve shape early on, it was hypothesized that the interaction of TATP vapors with metal-oxide sensors was not only attributed to heats of decomposition, but perhaps also to energies related to an adsorption-desorption process.

\subsection{Exploiting Chemisorption to Detect TATP Vapors}

Since chemisorption is associated with direct chemical bonding behavior between the adsorbate and the adsorbent, this opens up the possibility of identifying TATP through sensing these characteristic binding energies. Presumably, there should be a variation of binding energies between differing combinations of explosive vapors and adsorbent catalyst surfaces. By quantifying these energy effects among a variety of metal-oxides exposed to TATP, it may be possible to create an identification protocol for TATP (or other vapors) using known individual empirical sensor responses as the benchmark. The idea of using an array of sensors for screening of vapors is not a new concept, as this methodology is already being employed for other techniques, such as colorimetric sensing [8].

\subsection{Thermal Mass and Sensitivity}

Previously, solid-state sensors have been used as a thermodynamic sensing platform $[14,15]$. As this sensing technique relies on efficient sensing of heat effects, it would be beneficial to reduce the thermal mass of the sensor as much as possible. The 
solid-state sensor platform has a relatively large thermal mass which greatly limits sensitivity. The thin-film microheater on this platform also exhibits a relatively large noise floor, making successful detection of explosive vapors less obvious in real-time.

In this work, new low-mass sensors were investigated as the sensing platform and were sputter-coated with metal-oxide catalysts. These coil microheaters exhibited a much lesser noise floor, enabling these sensors to detect even the smallest energetic effects. The masses of these sensors were orders of magnitude less than the mass of the solid-state platform. Given the large reduction in thermal mass, sensing those thermodynamic effects related to decomposition, adsorption, and/or desorption were now possible. 


\section{CHAPTER 3}

\section{METHODOLOGY}

A thermodynamic sensor platform was previously developed for the detection of peroxide based and nitrogen based explosive vapors [14, 15]. This thermodynamic sensor measured the heat generated or absorbed by a metal-oxide catalyst in the presence of TATP, 2-4 DNT or ammonium nitrate. The technique employs a digital control system, which enables a microheater coated with a metal-oxide catalyst to be scanned over a selected temperature range. The electrical power difference due to catalytic interaction (i.e. the power difference between an uncoated microheater and catalyst coated microheater in the presence of the explosive molecule) was measured as a function of temperature for a variety of metal-oxide catalysts. In the present work, the thermodynamic sensor is comprised of a free-standing nickel coil (25- $\mu \mathrm{m}$ in diameter), which was sputter-coated with various metal-oxides. The coils were welded to four copper electrodes (HPI Inc., Ayer MA) and soldered to DB9 connectors. Metal-oxides including $\mathrm{SnO}_{2}, \mathrm{CuO}, \mathrm{ZnO}$ and $\mathrm{WO}_{3}$ were sputter-coated onto the nickel coils using an RF sputtering technique to ensure complete coverage of the surface. An MRC Model 8667 sputtering machine using an RF power of $200 \mathrm{~W}$ and a forward voltage of 800 volts was used to prepare the metal-oxide films. Table 3.1 shows the specific RF sputtering conditions used to deposit the metal-oxide catalysts for use in the thermodynamic sensor platform. 
Sputtering conditions for various metal oxide catalysts

\begin{tabular}{lllll}
\hline & Catalyst & & \\
\cline { 2 - 5 } & CuO & ZnO & WO3-TiO2 & SnO2 \\
\hline Target Diameter (mm) & 150 & 150 & 150 & 150 \\
Target material & $\mathrm{CuO}$ & $\mathrm{ZnO}$ & $\mathrm{W}-\mathrm{Ti}(90 / 10)$ & $\mathrm{SnO} 2$ \\
Power density (W/cm2) & 1.13 & 1.13 & 1.13 & 1.13 \\
Voltage (V) & 700 & 600 & 700 & 700 \\
Gas pressure (mTorr) & Ar. 7 & Ar. 9 & Ar. 6.3 O2: 0.7 & Ar. 7 \\
\hline
\end{tabular}

Table 3.1 RF sputtering conditions used to prepare the various metal-oxides used as catalysts.

A schematic of the apparatus used for the detection of explosive vapors is shown in Figure 3.1. At the inlet of the electronic trace detection system, compressed dry air is fed to an Alicat Scientific mass flow controller (MFC) set at a predefined constant flow rate. The incoming air stream flows to two manual valves adjusted such that all airflow initially passes through the empty loading chamber. To begin an explosive vapor test, these valve positions are switched simultaneously such that all airflow diverts from flowing through the empty chamber to flowing through the explosive-loaded chamber. Two additional Alicat Scientific MFCs were used to measure the output flow rates downstream to chambers containing the microheaters. One chamber contained a catalystcoated microheater, and the other contained a bare microheater which was used as a reference. The bare nickel microheater was not responsive to TATP or other explosives used in this study, and thus was used as a reference to subtract any false-positives such as sensible heat effects. Only the metal-oxide catalyst-coated coil is the "active" sensor element and responds to the explosive molecule of interest.

A digital control system employing a LabView program was used to heat the microheaters to a series of predetermined set point temperatures. The temperatures selected were based on the temperature coefficient of resistance (TCR) of the nickel coils, 
which was independently calibrated. The reference and catalyst-coated coils were held at constant electrical resistance (corresponding to a specific temperature) for about five minutes to minimize signal drift. After stabilization, data collection was initiated and the manual valves were simultaneously adjusted such that all gas flow was diverted from (initially) the empty loading chamber to the chamber containing the explosive analyte. The average amount of heat generated or absorbed by the catalyst in the presence of the target gas was measured as the change in electrical power required to maintain the catalyzed coil at a constant temperature. Typical experiments take several minutes to complete, since it can take that long for the sensor to reach its peak power level, i.e. the time it takes to reach equilibrium at each new temperature set point, which depends on the response to a specific analyte. It can also take a few minutes for the sensor output to return to its original baseline at the end of a test run, once the analyte supply valve is closed.

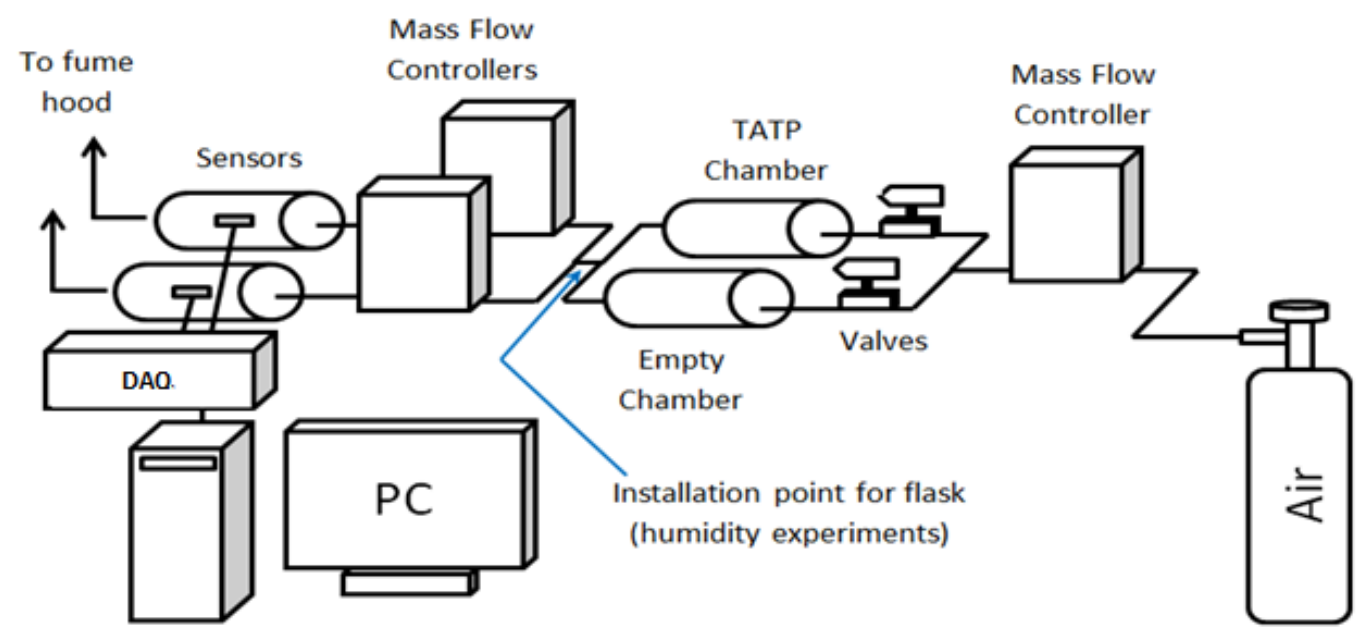

Figure 3.1: Schematic of the test bed used for thermodynamic sensor testing. Compressed air is fed to manual valves which are set to control the flow rates of carrier gas and TATP. Carrier gas laden with TATP flows downstream to the catalyst coated sensor and reference sensor. 
In addition, several experiments were performed over a range of sensor temperatures and flow rates to determine their effect on sensor selectivity and sensitivity. If sensor performance is not affected by high flow rates for example, such information would be useful for field applications that would likely require very fast sampling rates. These additional experiments were performed using the standard two-chamber protocol and simply varying the inlet MFC flow rate at the start of a test run. Humidity tests were also performed at constant temperature and flow rate to determine the effect (if any) of humidity on sensor response to TATP. To perform the humidity experiments, a sealed 500mL flask containing water (or appropriate salt solution) was located downstream from the TATP loading chamber (indicated by the blue arrow in Figure 3.1). Dry air was used as the carrier gas once again and the analyte stream (laden with moisture) flows from the headspace to the thermodynamic sensors.

Raw data was collected by LabView software as plots of Power (W) versus Time (s) and exported to Microsoft Excel for analysis. All data reported in this work as Power (W) versus Time (s) are the actual raw data sets obtained individually from LabView software. Sensor response magnitudes are reported as "Power Change (W)" - the overall power increase (or decrease) from their initial baseline power outputs to their new steadystate power outputs upon exposure to explosive vapors. This type of reporting was used particularly for sensitivity/selectivity analyses, with the goal of determining the magnitude of steady-state power supply changes with respect to the catalyst and/or explosive analyte chosen. Sensitivity/selectivity analyses were performed at constant flow rate and humidity settings and with varied temperature settings (from low to high temperature). Magnitudes of observed power changes were reported with respect to these varied sensor temperatures 
to determine its effect on sensor sensitivity among catalysts. Humidity experiments were performed under constant high temperature $\left(500^{\circ} \mathrm{C}\right)$ and constant flow rate $(250 \mathrm{sccm})$ settings. Flow rate experiments were also performed at high temperature $\left(500^{\circ} \mathrm{C}\right)$ and used dry air as the carrier gas. Catalyst microstructure of a used $\mathrm{SnO}_{2}$-coated coil sensor was analyzed using Scanning Electron Microscopy (SEM) in backscattered mode. 


\section{CHAPTER 4}

\section{FINDINGS}

Figure 4.1 shows the response (power increase) of a thermodynamic sensor exposed to $20 \mathrm{ppm}$ TATP, when the sensor was operated at a temperature of $500^{\circ} \mathrm{C}$. The response (power curve) went from baseline $(0.935 \mathrm{~W})$ to $0.955 \mathrm{~W}$ or nearly $20 \mathrm{~mW}$ higher than baseline, suggesting that the heat effect was endothermic in nature. Here, a net cooling effect was observed where additional power was supplied to the sensor to maintain the sensor temperature at the $500^{\circ} \mathrm{C}$ set point. The lag time associated with the thermodynamic response shown in Figure 4.1 was attributed to the time it takes to deliver the analyte to the sensor, which is a function of plumbing. When the supply of TATP to the sensor was "shut off", the sensor exhibited a slow return to baseline, which occurred at a linear rate and took several minutes to complete. Since the power level does return to the original baseline value, it suggests that the sensor response is completely reversible. The parabolic behavior of the power increase observed suggests that the response was cumulative with respect to time; i.e. instead of an instantaneous increase in power to a new peak level, the power curve rose slowly to a new peak value. The time for the signal to return to baseline after the $20 \mathrm{~mW}$ excursion was approximately 4 minutes. 


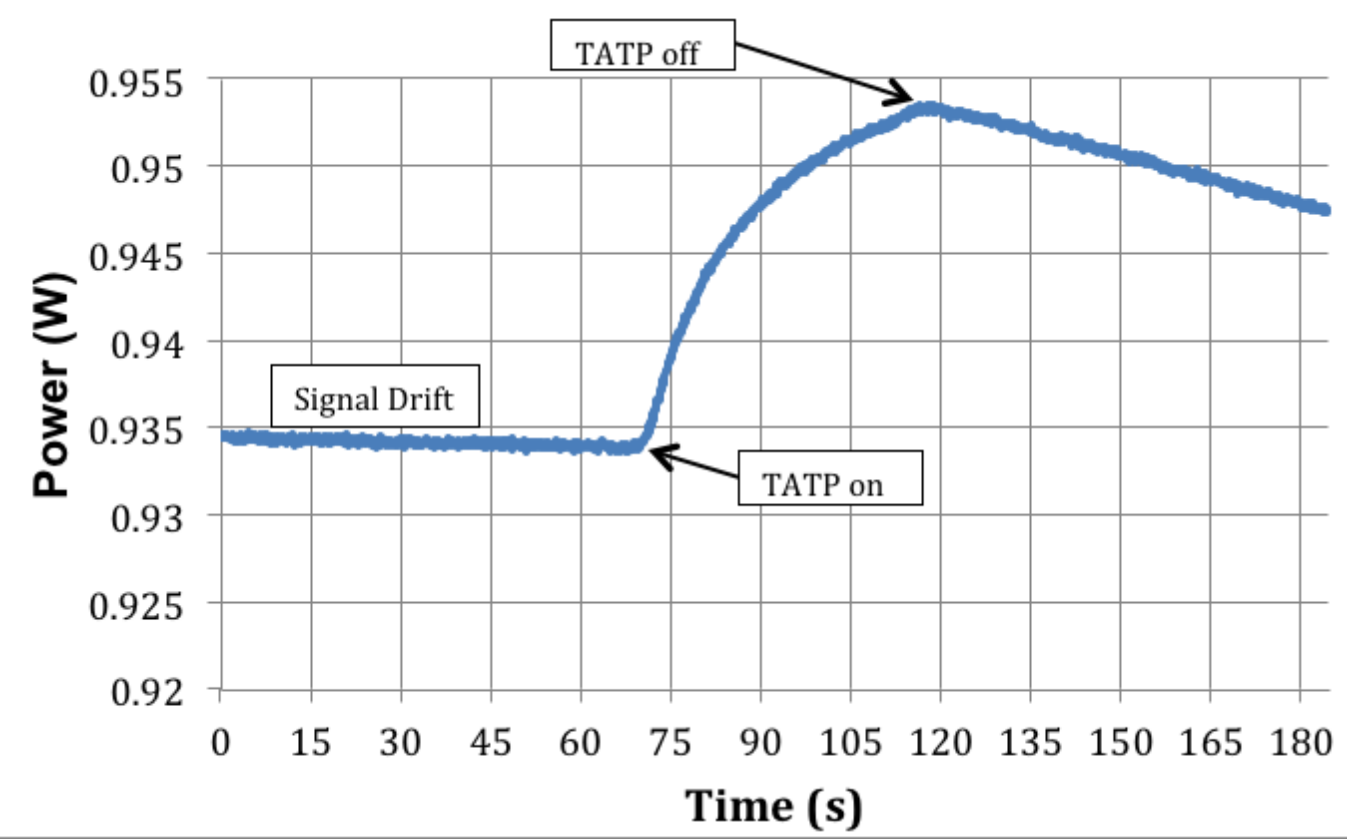

Figure 4.1: Response of a $\mathrm{SnO}_{2}$ coated coil $\left(500^{\circ} \mathrm{C}\right)$ sensor exposed to $20 \mathrm{ppm}$ TATP. Response (power increase) was $20 \mathrm{~mW}$ when the TATP supply was shut off. Note the slight drift in the baseline prior to the introduction of TATP.

Figure 4.2 shows the response of a thermodynamic sensor fabricated with a $\mathrm{SnO}_{2}$ catalyst cycled to $75^{\circ} \mathrm{C}, 100^{\circ} \mathrm{C}, 150^{\circ} \mathrm{C}, 250^{\circ} \mathrm{C}, 350^{\circ} \mathrm{C}, 450^{\circ} \mathrm{C}$ and $500^{\circ} \mathrm{C}$ and exposed to $20 \mathrm{ppm}$ TATP. The data was normalized with respect to the $75^{\circ} \mathrm{C}$ curve so that the relative power changes from a common baseline could be observed. Here, the power increased monotonically with sensor operating temperature, with the largest responses being observed at the highest operating temperatures. The peak responses here varied between $20 \mathrm{~mW}$ and $40 \mathrm{~mW}$ as the sensor temperature was varied from $75^{\circ} \mathrm{C}$ to $500^{\circ} \mathrm{C}$. The $\mathrm{SnO}_{2}$ catalyst-coated sensor cycled to $500^{\circ} \mathrm{C}$ exhibited the largest response to TATP vapor of any sensor investigated to date and the rate of power increase (slope of the response curve upon initial exposure to TATP) also varied with sensor operating temperature. Higher temperatures yielded the highest rate of power increase and the 
lower temperatures yielded the lowest rate of power increase, as might be expected for a rate controlled process.

The power curves shown in Figure 4.1 and Figure 4.2 resemble Langmuir adsorption isotherms [20, 21, 38, 39]. Adsorption isotherms typically show the dependency of surface coverage on adsorbate concentration, and as such, the behavior shown in Figure 4.1 and Figure 4.2 suggests that chemisorption or a related monolayeradsorption mechanism may be responsible for the observed behavior of our thermodynamic sensors. The rate of power increase (or slope of the response curve upon exposure to TATP) with increasing temperature shown in Figure 4.1 and Figure 4.2 supports a chemisorption/adsorption mechanism or related rate controlled process. To determine the activation energy for this rate-controlled process, an Arrhenius plot of the parabolic rate constants from Figure 4.2 were plotted as a function of sensor temperature (Figure 4.3). From the Arrhenius plot, an activation energy of $35 \mathrm{~kJ} / \mathrm{mol}$ was determined using linear regression analysis. An estimate of the corresponding activation energy for desorption was also calculated, when the TATP vapor supply was "shut off". Here, a linear rate constant was assumed and this linear rate constant increased with increasing temperature. The activation energy for this power decrease (desorption step) was 50 $\mathrm{kJ} / \mathrm{mol}$. 


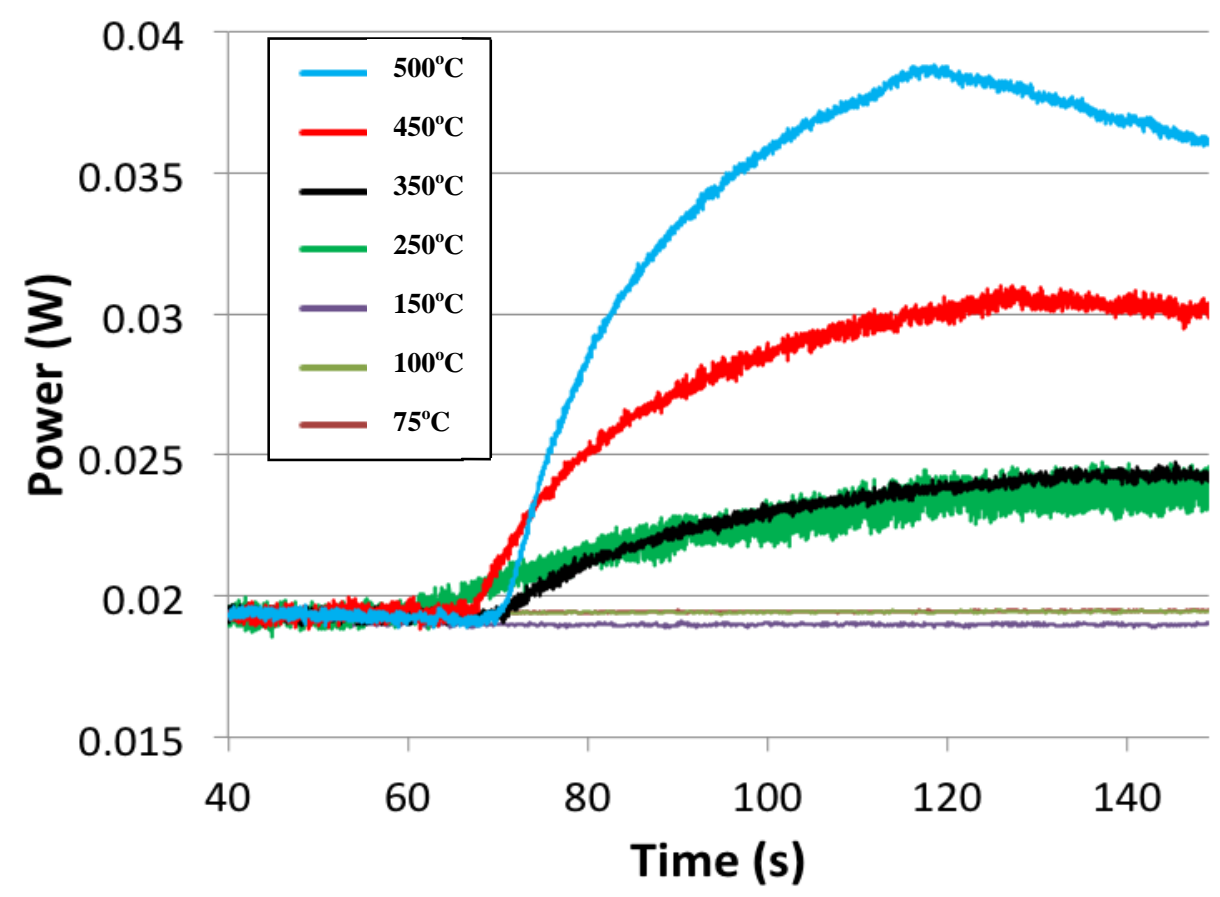

Figure 4.2: Response of a $\mathrm{SnO}_{2}$ coated-coil sensor held at $75^{\circ} \mathrm{C}, 100^{\circ} \mathrm{C}, 150^{\circ} \mathrm{C}, 250^{\circ} \mathrm{C}, 350^{\circ} \mathrm{C}, 450^{\circ} \mathrm{C}$ and $500^{\circ} \mathrm{C}$ exposed to $20 \mathrm{ppm}$ TATP.

Based on previous work, the decomposition of TATP by metal-oxides occurred by a catalytic pathway $[14,15]$, rather than a thermal pathway $[18,19]$. This suggests direct chemical bonding behavior with the surface must be taking place to some extent. In addition, the process appeared to be reversible, which rules out the possibility that any permanent chemical changes are taking place on the sensor surface itself. This further suggests that the metal-oxide surface is indeed acting as a catalyst, as opposed to a reactant. It can also be seen in Figure 4.2 that some level of thermal activation is required in order to observe any obvious power response behavior above the noise floor. This leaves several possibilities for what exactly the previous calculated activation energies may be actually representative of in the larger picture, especially given that monolayer adsorption-desorption behavior is likely the apparent observation. The activation energies calculated for these observed detection (uphill and downhill) 
processes could possibly be attributed to various steps of activated chemisorption (see Figure 2.3). These may include the activation energy barrier to desorb explosive analyte/breakdown products from the sensor $\left(\mathrm{E}_{\mathrm{des}}\right)$, the activation energy barrier between physisorbed and chemisorbed states of analyte $\left(E_{a}\right)$, the net activation energy barrier between the initial (zero) energy state of analyte vapors and the chemisorbed state ( $\left.E_{\text {act }}\right)$ or a summation of all activation energies related to an entire activated chemisorption/desorption pathway.

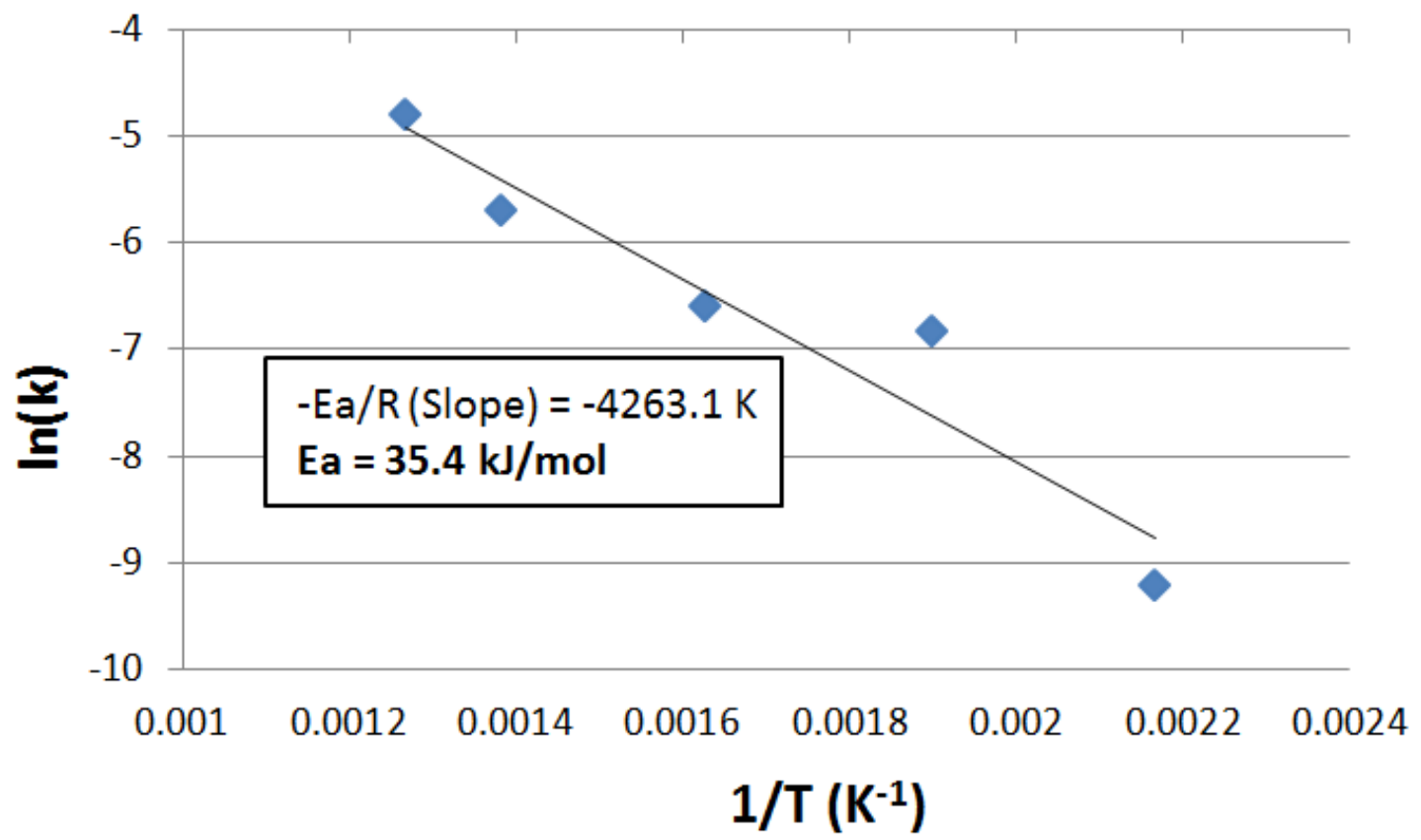

Figure 4.3: Arrhenius plot of the parabolic rate constant as a function of temperature for a $\mathrm{SnO}_{2}$ sensor exposed to TATP. The activation energy calculated over the temperature range of interest (Figure 4.2 "uphill” response) was approximately $35.4 \mathrm{~kJ} / \mathrm{mol}$.

It was also hypothesized that the calculated activation energies may shed some light on whether or not the observed process was related to physisorption or chemisorption under the assumption that the energy calculated represents a binding energy instead ( $E_{a d s}$, or $E_{d}$, Figure 2.3). However, the activation energy values 
approximated above for these experiments $(35 \mathrm{~kJ} / \mathrm{mol})$ fall near the border between physisorption and chemisorption binding energies, which are broadly defined to be about $48 \mathrm{~kJ} / \mathrm{mol}$ using the $0.5 \mathrm{eV} /$ molecule basis [20]. The activation energy magnitudes alone are not enough to explain the observed phenomena, given that there are clearly multiple possible explanations for what energies specifically are being "sensed" by these sensors.

Previously, for example, it was suggested that the heat effects could be strictly related to heats of decomposition of TATP $[14,15]$. That could be true, or only partially true if these other proposed activation energy-barriers are also directly contributing to the effect. The activation energy obtained above $(35.4 \mathrm{~kJ} / \mathrm{mol})$ is much lower than that for the activation energy for thermal decomposition of TATP, which is approximately 150 $\mathrm{kJ} / \mathrm{mol}$ [40]. However, it is very close to the activation energy for thermal decomposition of hydrogen peroxide [41], which suggests that the mechanism could first be thermal decomposition of TATP away from the sensors surface to produce hydrogen peroxide as a byproduct, followed by a second decomposition of hydrogen peroxide catalyzed by the metal-oxide surface.

The responses using $\mathrm{SnO}_{2}$ are clearly very endothermic responses with respect to the analyte - that is, energy is abstracted from the sensor to the analyte (a net "cooling" effect), resulting in an increase in power supply over time to maintain sensor temperature. Large activation barriers would only exacerbate this endothermic effect should they be overcome (as they are also endothermic in nature), and also hinder the process if insufficient energy is supplied to surpass the barrier. As previously stated, a thermal activation barrier has been observed which, according to Figure 4.2, appears to be 
overcome somewhere between $150^{\circ} \mathrm{C}$ (no response) and $250^{\circ} \mathrm{C}$ (weak, but noticeable response). The initial activation barrier of an activated chemisorption process ( $E_{a}$ from physisorbed state or $E_{\text {act }}$ from zero-state) might be what is overcome at higher temperatures, resulting in subsequent detectable heat effects at the surface related to direct chemical bonding behavior. The exact mechanism taking place at the surface is still unknown and up for speculation.

At high enough temperatures (observed $250^{\circ} \mathrm{C}$ and higher) and after $\sim 60 \mathrm{sec}$ exposure, the power curves shown in Figure 4.2 increased to new steady-state values. At these new steady-state values, it is likely that adsorption/desorption processes reached an equilibrium or quasi-equilibrium state, such that the adsorption of fresh molecules was dependent on a slower, rate- determining step once saturation occurred. It is hypothesized that at these higher temperatures, the explosive vapor adsorbs relatively quickly on the catalyst surface, but thermally desorbs over a much longer time period; i.e. desorption is a slower, endothermic process and as such is the rate-determining step. The adsorbate must overcome a large activation barrier to desorb from the surface, which is supplied by thermal energy of the sensor.

If trace detection is somehow related to chemisorption, it is not surprising that higher temperatures yielded larger sensor responses. At higher temperatures, more thermal energy is available to drive the thermal desorption process; i.e. they are desorbed much more readily due to sufficient heat supply. As the number of adsorption-desorption cycles for a given analyte-catalyst interaction were increased, larger cumulative heat effects were observed. This behavior was observed for the catalyst-coated sensors in the 
form of a progressively larger power increase with respect to time at higher temperatures in order to maintain the predetermined temperature set points.

To determine the selectivity of the low-mass thermodynamic sensors, a number of different metal-oxide catalysts were evaluated in terms of sensor performance. Figure 4.4 shows the power change as a function of sensor temperature for 5 different metal-oxide catalysts exposed to $20 \mathrm{ppm}$ TATP. The response using $\mathrm{SnO}_{2}$ catalysts was greater than any of the metal-oxides catalysts evaluated to date. Thus, Figure 4.4 clearly demonstrates sensor selectivity in that sensor response to TATP varied widely with catalyst. For example, a negative response was observed for the $\mathrm{CuO}$ catalyst whereas the other oxides exhibited a positive response. The negative response may be attributed to an exothermic desorption process, rather than the endothermic process observed for other catalysts - and it has been noted in literature that metals such as copper and silver may exhibit this effect due to their large work function [42]. These variations in TATP response show that there are clear differences in sensor selectivity for TATP among the oxides, with $\mathrm{SnO}_{2}$ exhibiting the greatest affinity for TATP vapors relative to the other oxide catalysts. Sensor selectivity was also demonstrated in Figure 4.5 where the power change of a tin oxide coated sensor as a function of temperature is shown for three different explosives (TATP, 2,4-DNT and ammonium nitrate). $\mathrm{SnO}_{2}$ coated sensors exhibited much greater selectivity for TATP relative to the nitrogen-based explosives 2,4-DNT and ammonium nitrate. Two different $\mathrm{SnO}_{2}$ coating thicknesses were used to demonstrate the effect of catalyst coverage on sensor response (Figure 4.4). The $\mathrm{SnO}_{2}$ catalyst sputtered for two hours had much better coverage than the one sputtered for only one hour, as evidenced by the greater response. As observed, the greater the surface coverage, the greater number 
of available adsorption sites for TATP interaction and the larger the response. All other catalyzed sensors $(\mathrm{ZnO}, \mathrm{WO}, \mathrm{NiO}$ and $\mathrm{CuO})$ were sputtered for 2 hours to ensure that the coil microheaters were conformally coated with catalyst material.

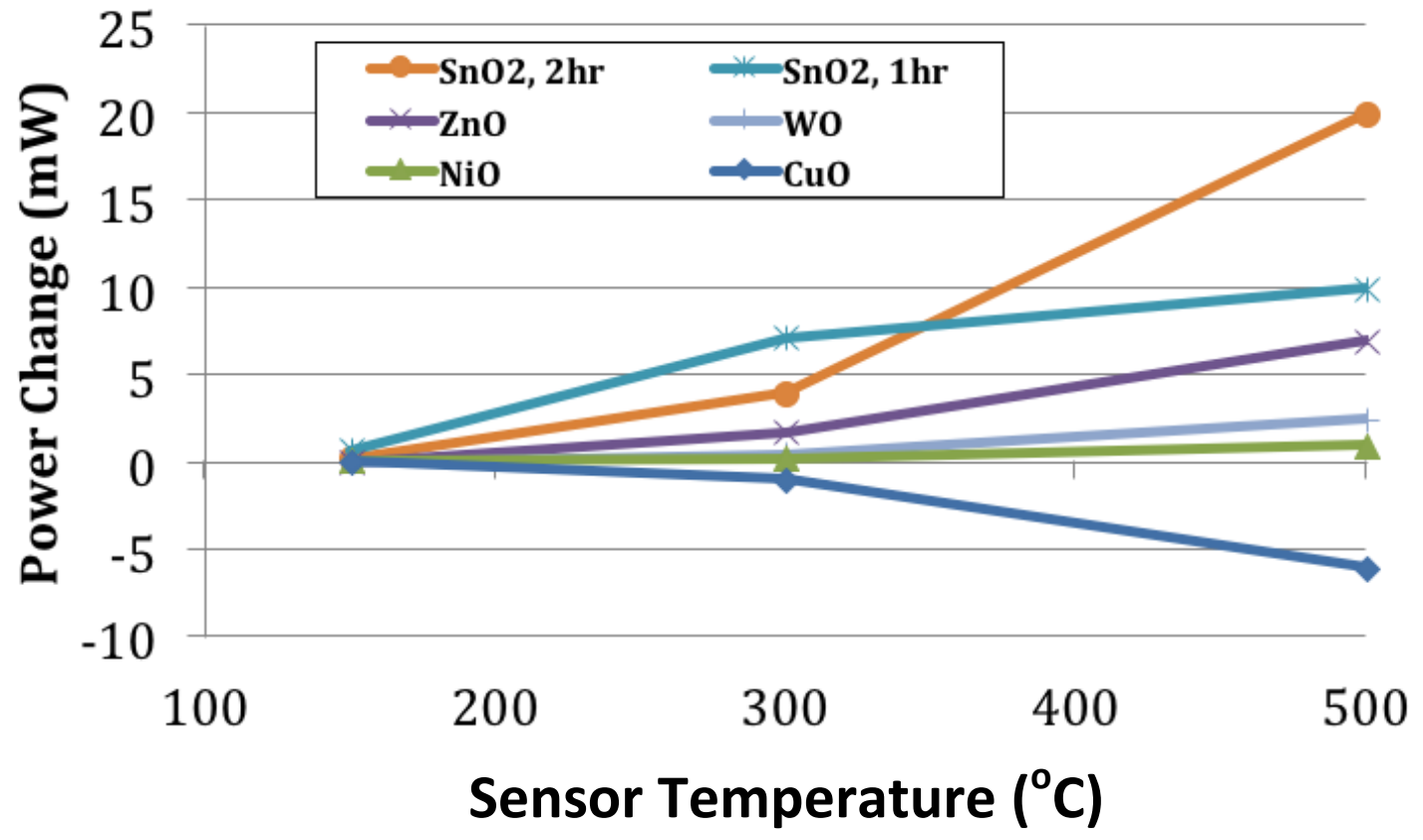

Figure 4.4: Sensor selectivity to TATP for a variety of sputtered metal-oxide catalysts. $\mathrm{SnO}_{2}$ was the most sensitive catalyst to TATP vapor. Note that $\mathrm{CuO}$ exhibited a negative power change whereas all other catalysts exhibited a negative power change upon exposure to TATP. All catalysts were sputtered for 2 hours with the exception of one $\mathrm{SnO}_{2}$ sensor which was sputtered for 1 hour. 


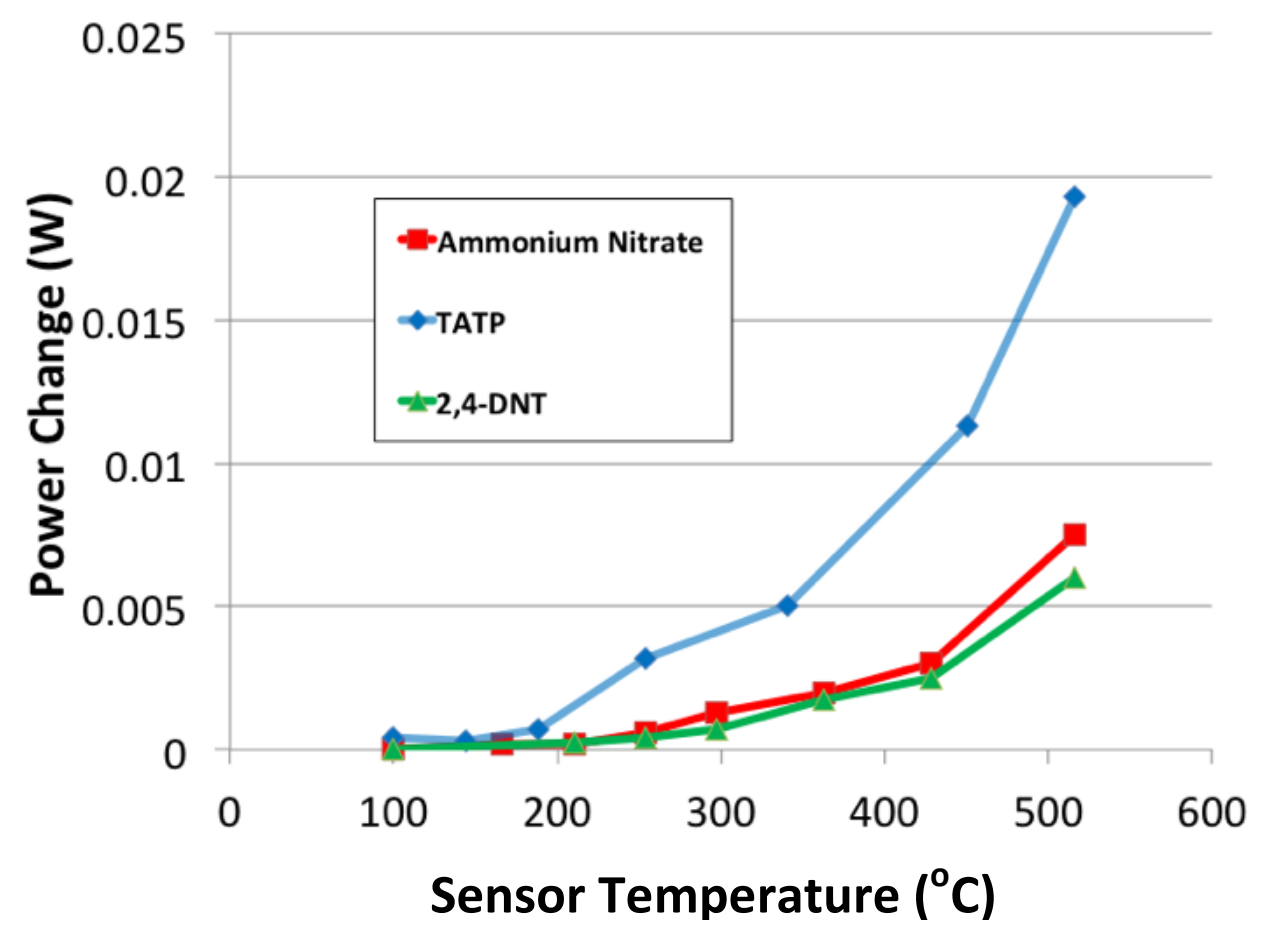

Figure 4.5: Sensor response (power change) as a function of temperature for a $\mathrm{SnO}_{2}$ catalyst coated sensor exposed to TATP, 2,4 DNT and ammonium nitrate. $\mathrm{SnO}_{2}$ coated sensor exhibited much greater selectivity for TATP relative to the nitrogen-based explosives; 2,4-DNT and ammonium nitrate.

Figure 4.6 shows the effect of sampling rate (flow rate) on sensor sensitivity. Here, flow rate was varied from $50 \mathrm{sccm}$ to $500 \mathrm{sccm}$ and the peak power increased by approximately $6 \mathrm{~mW}$ as the mass flow rate was increased from $50 \mathrm{sccm}$ to $250 \mathrm{sccm}$. There was no further change in sensor response once a flow rate of $250 \mathrm{sccm}$ was achieved. There were no adverse effects on sensor performance when higher flow rates were used. Flow rate is considered to be an important parameter when testing sensors in the field, since the flow rate may have to be adjusted to sample the desired volume of air in a reasonable time for screening purposes. A more sluggish response (on the order of minutes) was observed under low flow rate conditions $(50 \mathrm{sccm})$ whereas higher flow rate conditions produced a response time of $\sim 30$ seconds. Therefore, the use of higher flow rates not only improves sensor sensitivity but also reduces the response time as well. 


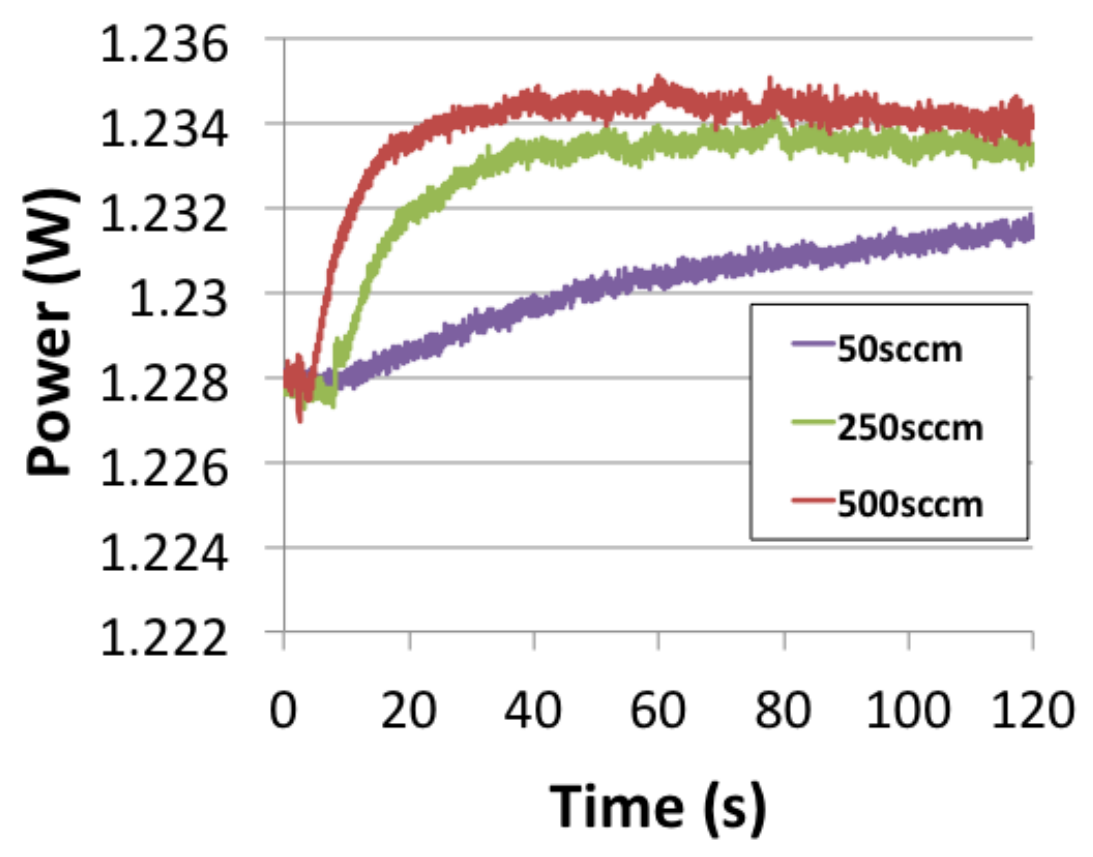

Figure 4.6: Responses of a $\mathrm{SnO}_{2}$ coated coil sensor to TATP as a function of volumetric flow rate. Volumetric flow rates ranging from $50 \mathrm{sccm}$ to $500 \mathrm{sccm}$ were investigated when the sensor temperature was maintained at $500^{\circ} \mathrm{C}$.

Humidity test results confirmed that humidity does influence the performance of the low-mass thermodynamic sensors, as shown in Figure 4.7. Specifically, sensor response decreased by approximately $65 \%$ when exposed to high humidity conditions $\left(100 \% \mathrm{RH}\right.$ at $\left.77^{\circ} \mathrm{F}\right)$. This degradation was slightly reduced when the relative humidity was reduced to the $15-75 \%$ level, as shown in Figure 4.7. Under moderate relative humidity conditions, even though the signal somewhat diminished, the response to 20 ppm TATP was clearly discernable from the noise floor. Some of the decrease in sensor response (power) was due to the much larger headspace in the vessel that contained water at room temperature, over which the carrier gas containing the explosive vapor was passed. The standard two-chamber protocol (Figure 3.1) utilizes a very small headspace relative to the headspace in the chamber used to generate the desired humidity levels. 
Therefore, the concentration of TATP in the empty vessel headspace (red curve in Figure 4.7) was considerably more dilute than the standard two-chamber protocol (blue curve in Figure 4.7). The volume of the vessel used to generate the desired humidity level is about 40 times larger than that of the chamber where the solid TATP is loaded. Also worth noting is the difference in response time for the empty vessel condition versus the standard protocol. Here, the peak response for the empty vessel condition was attained after 200s, as compared to 40s without the dilution effect.

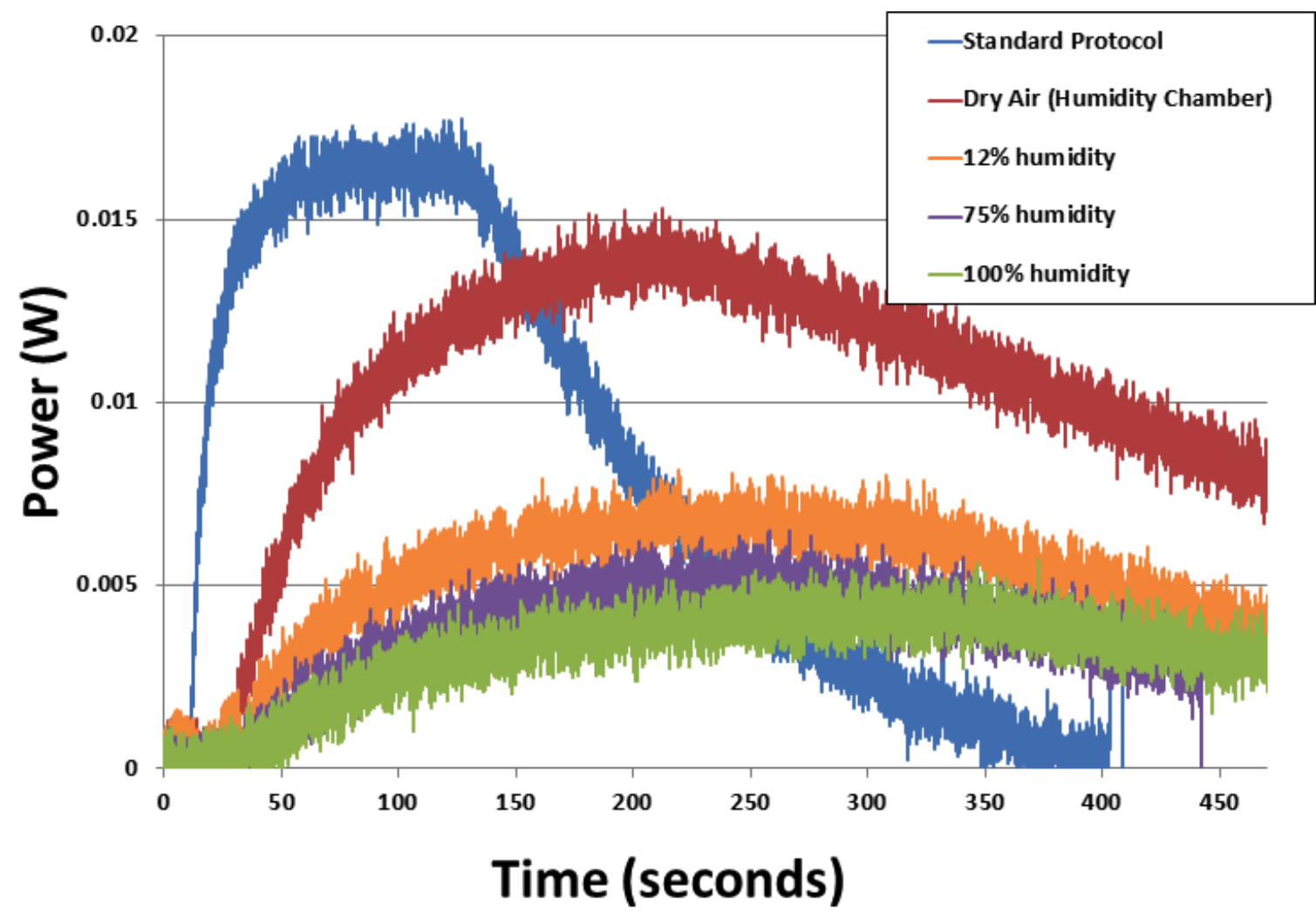

Figure 4.7: Low-mass thermodynamic sensor response to $20 \mathrm{ppm}$ TATP at $500^{\circ} \mathrm{C}$ as a function of relative humidity. An empty vessel downstream from the TATP chamber (blue curve) resulted in a lower response due to dilution effects (red curve). 
Figure 4.8 and Figure 4.9 show the microstructure of a tin oxide catalyst that was repeatedly cycled from room temperature to $500^{\circ} \mathrm{C}$. These tin oxide coatings exhibited relatively smooth rounded protuberances with minimal surface area, typical of an assputtered film (Figure 4.8). After repeated exposure to TATP at $500^{\circ} \mathrm{C}$, there was no apparent change in the microstructure of the tin oxide catalyst. However, in Figure 4.9, the columnar structure evident in the cross section of the tin oxide coating indicated that the layer closest to the nickel surface was slightly lighter in color compared to the outer layer of the coating. Since these SEMs were taken in backscattered mode, the contrast developed in these images is dependent on atomic number of the atoms comprising the coating. The darker outer layer in the coating cross section of the micrograph of Figure 4.9 suggests that this material may contain more oxygen and less metal than the inner layer. Also noteworthy is that the tin oxide coating in Figure 4.9 has separated from the nickel and led to the formation of a noticeable gap between the tin oxide coating and nickel surface. This is likely due to thermal stresses developed in the coating as a result of thermal cycling, which produced a number of micro-cracks in the coating. Catalytic activity may be enhanced in the vicinity of this gap, but additional investigation is required to see how this affects performance of these low-mass thermodynamic sensors. 


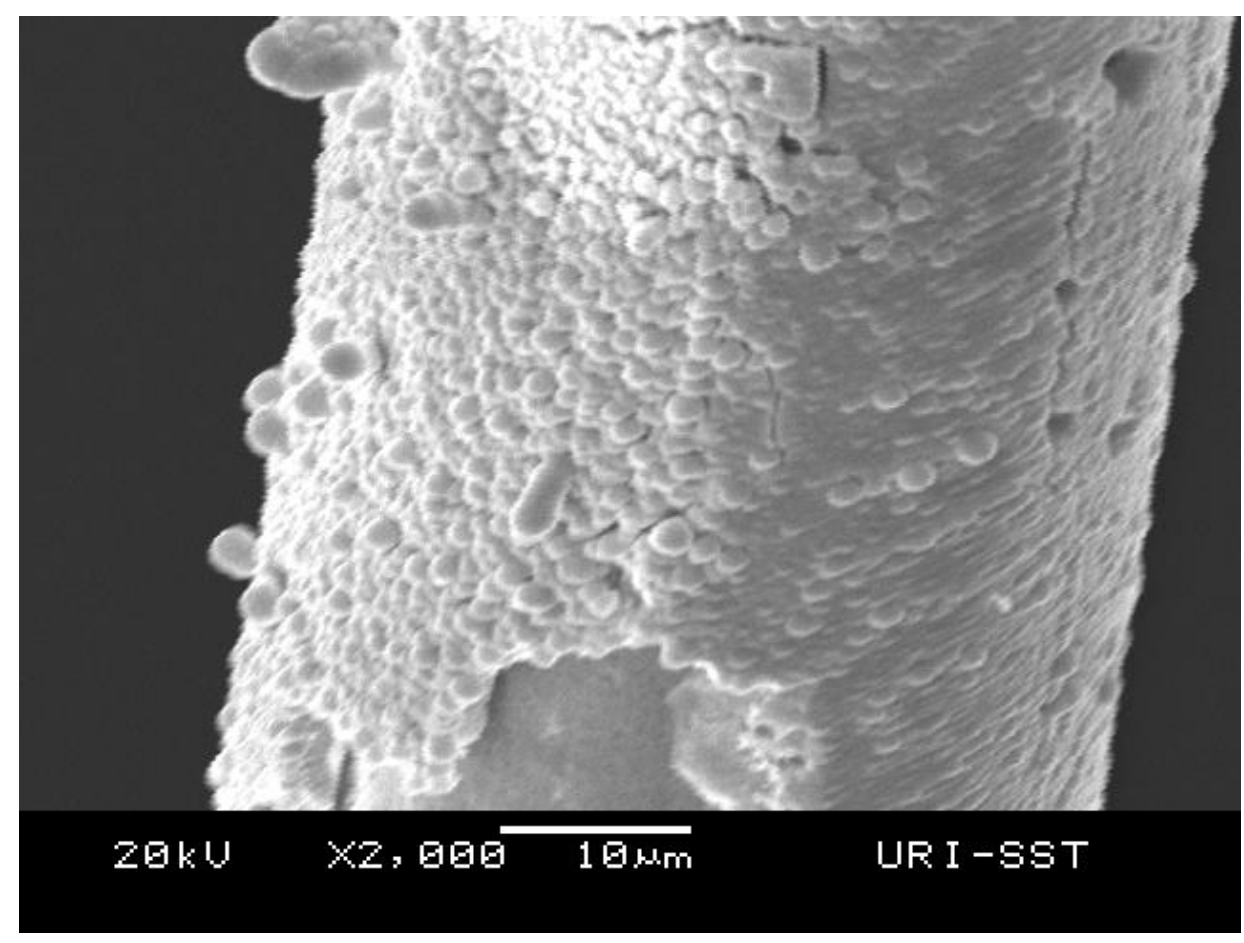

Figure 4.8. SEM micrograph of a tin oxide coated coil after repeated thermal cycling to $500^{\circ} \mathrm{C}$ Micrograph was taken in backscattered mode.

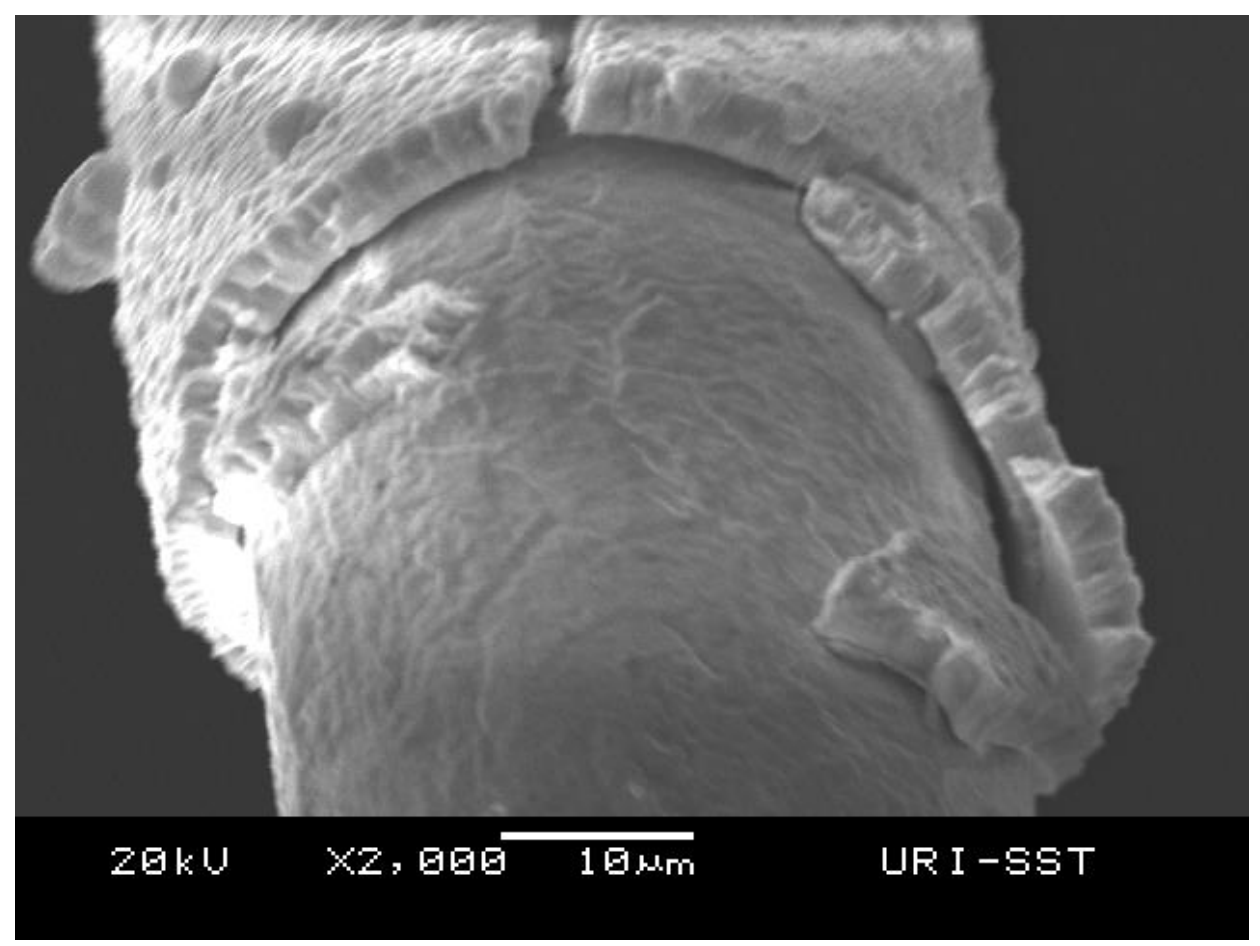

Figure 4.9. SEM micrograph of a tin oxide coated coil after repeated thermal cycling to $500^{\circ} \mathrm{C}$. Micrograph was taken in backscattered mode highlighting the dark and light layers in the coating cross section. 


\section{CHAPTER 5}

\section{CONCLUSION}

A low-mass thermodynamic sensor capable of detecting TATP at 20ppm was demonstrated. Ultrafine coil microheaters ( $25 \mu \mathrm{m}$ in diameter) were used to reduce the thermal mass of previously developed solid-state sensors by a factor of 185 . This lowmass thermodynamic sensor platform exhibited the sensitivity (power increases as high as $20 \mathrm{~mW}$, with a low noise floor), selectivity (range of responses between $-10 \mathrm{~mW}$ to $+20 \mathrm{~mW}$ among different catalysts) and response time (30-60s) necessary for the continuous monitoring of TATP at trace levels. $\mathrm{SnO}_{2}$ catalysts proved to be the most responsive of all of the metal-oxides investigated to date for the detection of TATP $\left(20 \mathrm{~mW}\right.$ power increase at $500^{\circ} \mathrm{C}$ ). In addition, the $\mathrm{SnO}_{2}$ coated coils exhibited the greatest selectivity for TATP relative to nitrogen-based explosive vapors, namely 2,4DNT and ammonium nitrate. This was evidenced by $\mathrm{SnO}_{2}$-catalyzed sensor responses to TATP vapors (power increases) which were over twice the magnitude of responses obtained for ammonium nitrate and 2,4-DNT vapors at the same temperatures. No detrimental effects were observed when the sampling rate (flow rate) was increased by an order of magnitude (from $50 \mathrm{sccm}$ to $500 \mathrm{sccm}$ ) and in fact, the response time decreased and peak response actually increased when sampling rates above $250 \mathrm{sccm}$ were employed. Even though the response of the sensor to trace amounts of TATP was somewhat degraded under moderate to high humidity conditions (by over 50\%), the signal was still strong enough to be discernable from the noise floor or background. The detection of explosive vapors based on the thermodynamic effects presented here may not only be related to the catalytic decomposition of the energetic molecules but may also be 
related to activated chemisorption, as a thermal activation energy barrier was observed to be surpassed near a sensor temperature of $250^{\circ} \mathrm{C}$, and Langmuir-type kinetics were observed. The observed chemical specificity amongst metal-oxide catalysts and explosive vapors also supports the possibility of chemisorption-related behavior being involved. Thus, detection may be related to a combination of mechanisms including catalytic decomposition of TATP combined with activated chemisorption and desorption. In addition, the results presented here suggest that by reducing the thermal mass of the thermodynamic sensor even further, e.g. by moving to a MEMS-based sensing platform where the microheater is fabricated directly onto $\mu \mathrm{m}$-thick free standing diaphragms, the detection limits could be lowered even further. Low-mass sensors with the capabilities described within could be deployed in an array format in closed spaced venues for screening purposes where the public may be exposed to potential threats from IEDs employing TATP as the triggering device. 


\subsection{Future Work}

Findings indicate that real-time trace detection of TATP is feasible with a lowmass thermodynamic sensor using the described laboratory protocol under typical environmental conditions. Looking forward, one goal is to develop a portable version of the detection system and determine if TATP can be detected in the field.

In addition, the improvement in sensor sensitivity using low-mass sensors suggests that the detection limits of TATP could be dropped even lower if a sensor with less mass than the coil sensors (such as a MEMS platform) is used in the future. Prototyping a MEMS sensor array, if possible, should be one of the next research priorities for improving the sensitivity and response time for thermodynamic TATP detection.

Other improvements to maximize the number of TATP interactions with the sensor surface per unit mass of the sensor should be considered, such as the use of nanostructured catalysts [43]. These have not been used on the low-mass coil sensors yet and may prove to help bolster their sensitivity. Given that the present sensor behavior using sputtered catalysts appears to be attributed to an adsorption-desorption process, increasing the total number of adsorption sites through increased catalyst surface area should significantly increase the magnitude of all sensor responses to better distinguish them from the noise floor, especially since the noise floor has been greatly reduced. This may lower the detection limits of TATP further.

Interference testing will also be required going forward to ensure that TATP may be selectively detected by low-mass thermodynamic sensors through materials such as 
external packaging, and to ensure that characteristic TATP responses are not obtained as false-positives by other vapor analytes.

Additional experiments to better prove out a potential chemisorption mechanism should also be considered, as this work observes the phenomena very indirectly at best (and thus cannot be used as concrete proof for a chemisorption mechanism). Techniques such as He Diffractive Scattering, Ultraviolet Photoemission Spectroscopy (UPS) and Metastable Deexcitation Spectroscopy (MDS) for example have demonstrated the ability to observe the structural and electronic properties of $\mathrm{Ag}(110)$ surfaces following dissociative chemisorption of oxygen [44]. Using a similar technique to observe the effects on metal-oxide surfaces upon exposure to TATP (or other vapors) may provide new insight into possible mechanisms of action for this sensing technology. 


\section{BIBLIOGRAPHY}

[1] M. S. Lee, "Analysis of Triacetone Triperoxide Explosive by Mass Spectrometry," Mass Spectrometry Handbook, Wiley-Blackwell, Oxford (2012) 373-88.

[2] G. A. Buttigieg, A. K. Knight, S. Denson, C. Pommier, M. B. Denton, "Characterization of the explosive triacetone triperoxide and detection by ion mobility spectrometry," Forensic Sci. Int. 135 (2003) 53-59.

[3] R. Rasanen, M. Nousiainen, K. Parakorpi, M. Sillanpaa, L. Polari, O. Anttalainen, M. Utriainen, "Determination of gas phase triacetone triperoxide with aspiration ion mobility spectrometry and gas chromatography-mass spectrometry," Anal. Chim. Acta 623 (2008) 59-65.

[4] J. Wilkinson, C. T. Konek, J. S. Moran, E. M. Witko, T. M. Korter, "Terahertz absorption spectrum of triacetone triperoxide (TATP)," Chem. Phys. Letters 478 (2009) 172-174.

[5] A. Stambouli, A. El Bouri, T. Bouayoun, M. A. Bellimam, "Headspace GC/MS detection of TATP traces in post-explosion debris," Forensic Sci. Int. 146S (2004) S191-S194. 
[6] C. Mullen, A. Irwin, B. V. Pond, D. L. Huestis, M. J. Coggiola, H. Oser, "Detection of explosives and explosives-related compounds by single photon laser ionization time-of-flight mass spectrometry," Anal. Chem. 78 (2006) 3807.

[7] C. Shen, J. Li, H. Han, H. Wang, H. Jiang, Y. Chu, "Triacetone triperoxide using low reduced-field proton transfer reaction mass spectrometer," Int. J. Mass Spectrometry 285 (2009) 100-103.

[8] H. Lin, K. S. Suslick, "A colorimetric sensor array for detection of triacetone triperoxide vapor," J. Am. Chem. Soc. 132 (2010) 15519-15521.

[9] Z. Li, W. P. Bassett, J. R. Askim and K. S. Suslick, "Differentiation among peroxide explosives with an optoelectronic nose," Chem. Communications 51 (2015) 15312.

[10] S. K. Mamo and J. Gonzalez-Rodriguez, "Development of a Molecularly Imprinted Polymer-Based Sensor for the Electrochemical Determination of Triacetone Triperoxide (TATP)," Sensors 12 (2004) 23269-23282.

[11] A. Lichtenstein, "Supersensitive fingerprinting of explosives by chemically modified nanosensors arrays," Nat. Communications 5 (2014) 4195-5195. 
[12] D. Mallin, "Increasing the Selectivity and Sensitivity of Gas Sensors for the Detection of Explosives." MS Thesis (2014) University of Rhode Island.

[13] Z. Caron, D. Mallin, M. Champlin, and O.J. Gregory, "A Pre-Concentrator for Explosive Vapor Detection," Electrochemical Society Transactions 66 (2015) 3844.

[14] M. Amani, C. Yun, K. L. Waterman, C. M. Hurley, M. J. Platek and O. J. Gregory, "Detection of triacetone triperoxide (TATP) using a thermodynamic based gas sensor," Sensors and Actuators B. 162 (2012) 7-13.

[15] Y. Chu, D. Mallin, M. Amani and O. J. Gregory, "Detection of Peroxides Using Pd/SnO2 Nanocomposite Catalysts," Sensors and Actuators; B Chemical 197 (2014) 376-384.

[16] J. Warmer, P. Wagner, M. J. Schöning, and P. Kaul, "Detection of triacetone triperoxide using temperature cycled metal-oxide semiconductor gas sensors," Phys. Status Solidi A 2126 (2015) 1289-1298.

[17] R. S. Ray, B. Sarma, S. Mohanty, and M. Misra, "Theoretical and experimental study of sensing triacetone triperoxide (TATP) explosive through nanostructured TiO2 substrate," Talanta 118 (2014) 304-11. 
[18] F. Dubnikova, R. Kosloff, J. C. Oxley, J. L. Smith, and Y. Zeiri, "Role of Metal Ions in the Destruction of TATP: Theoretical Considerations," Journal of Physical Chemistry A 11538 (2011) 10565-10575.

[19] F. Dubnikova, R. Kosloff, J. Almog, Y. Zeiri, R. Boese,|H. Itzhaky, X. Aaron and E. Keinan, "Decomposition of Triacetone Triperoxide is an Entropic Explosion," Journal of the American Chemical Society 1274 (2015) 1146-1149.

[20] K. Oura, V. G. Lifshits, A. A. Saranin, A. V. Zotov, M. Katayama, "Surface Science: An Introduction," Springer-Verlag (2003) 193-194, 295-323.

[21] R. A. Latour, "The Langmuir isotherm: A commonly applied but misleading approach for the analysis of protein adsorption behavior," Journal of Biomed Mater Res Part A 103A (2015) 949-958.

[22] M. Marshall, J. C. Oxley, “Aspects of Explosives Detection,” Elsevier (2011) 1126.

[23] P. Saukko, J. A. Siegel, "Improvised Explosive Devices," Encyclopedia of Forensic Sciences, Amsterdam: Elsevier, Academic (2013) 59-63.

[24] Y. Sun, "Field Detection Technologies for Explosives," UK: ILM Publications (2010) 1-90. 
[25] J. Liu, Y. Lu, "Response Mechanism for Surface Acoustic Wave Gas Sensors Based on Surface-Adsorption,” Sensors 14 (2014) 6844-6853.

[26] J. Yinon, "Counterterrorist Detection Techniques of Explosives," Elsevier B.V. (2007) 1-37.

[27] J. W. Gardner, J. Yinon, "Electronic Noses \& Sensors for the Detection of Explosives," Netherlands: Springer (2006) 53-69.

[28] M. Tourné, “Developments in Explosives Characterization and Detection," J Forensic Res 122 (2013).

[29] E. Sokol, R. J. Noll, R. G. Cooks, L. W. Beegle, H. I. Kim, et al., "Miniature mass spectrometer equipped with electrospray and desorption electrospray ionization for direct analysis of organics from solids and solutions.” International Journal of Mass Spectrometry 306 (2011) 187-195.

[30] A. M. Graichen, R. W. Vachet, "Multiplexed MS/MS in a miniature rectilinear ion trap,” J Am Soc Mass Spectrom 22 (2011) 683-688.

[31] J. S. Wiley, J. T. Shelley, R. G. Cooks, "Handheld low-temperature plasma probe for portable 'point-and-shoot' ambient ionization mass spectrometry," Anal Chem 85 (2013) 6545-6552.

[32] C. Janfelt, R. Graesboll, F. R. Lauritsen, "Portable electrospray ionization mass spectrometry (ESI-MS) for analysis of contaminants in the field," International Journal of Environmental Analytical Chemistry 92 (2012) 397-404. 
[33] W. Chen, K. Hou, X. Xiong, Y. Jiang, W. Zhao, et al., "Non-contact halogen lamp heating assisted LTP ionization miniature rectilinear ion trap: a platform for rapid, on-site explosives analysis,” Analyst 138 (2013) 5068-5073.

[34] S. Singh, "Sensors - An effective approach for the detection of explosives," Journal of Harzardous Materials 144 (2007) 15-28.

[35] S. Steinhauer, "Gas Sensing Properties of Metal Oxide Nanowires and their CMOS Integration," (Doctoral Dissertation) Retrieved from Vienna University of Technology Publication Database (2014).

[36] S. R. Morrison, “The Chemical Physics of Surfaces," New York: Plenum Press (1990).

[37] P. Atkins and J. de Paula, "Physical Chemistry," Oxford: Oxford University Press (2006).

[38] J. R. H. Ross, "Heterogeneous Catalysis: Fundamentals and Applications," Elsevier (2011) 17-45.

[39] J. H. de Boer, "The Dynamical Character of Adsorption," Oxford: Oxford University Press (1968) 60-61.

[40] J. Oxley, J. Smith, H. Chen, "Decomposition of a Multi-Peroxidic Compound: TATP," Propellants, Explosives, Pyrotechnics 27 (2002) 1-8.

[41] C. Lousada, M. Jonsson, "Kinetics, Mechanisms and Activation Energy of H2O2 Decomposition on the Surface of Zirconia," J. Phys. Chem. C 11425 (2010) 11202-11208. 
[42] J. H. de Boer, "Endothermic Chemisorption and Catalysis," Advances in Catalysis 9 (1956) 472-480.

[43] Z. Caron, "Novel Catalyst Development for Chemical Sensors,” MS Thesis (2016) University of Rhode Island.

[44] M. Canepa, C. Guarnaschelli, L. Mattera, M. Polese, S. Terreni, “An experimental apparatus to study chemisorption at surfaces by He diffractive scattering, ultraviolet photoemission, and metastable deexcitation spectroscopies," Review of Scientific Instruments 62 (1991), 1431. 